OPEN ACCESS

Edited by: Nicole Leclerc,

Université de Montréal, Canada

Reviewed by:

Katrin Deinhardt,

University of Southampton,

United Kingdom

Adriana Ferreira,

Northwestern University,

United States

${ }^{*}$ Correspondence:

Vivian Hook

vhook@ucsd.edu

Specialty section:

This article was submitted to

Neurodegeneration,

a section of the journal

Frontiers in Neuroscience

Received: 29 April 2021

Accepted: 09 September 2021

Published: 21 October 2021

Citation:

Boyarko B and Hook V (2021) Human Tau Isoforms and Proteolysis for Production of Toxic Tau Fragments

in Neurodegeneration.

Front. Neurosci. 15:702788.

doi: 10.3389/fnins.2021.702788

\section{Human Tau Isoforms and Proteolysis for Production of Toxic Tau Fragments in Neurodegeneration}

\author{
Ben Boyarko ${ }^{1}$ and Vivian Hook ${ }^{1,2 *}$ \\ 1 Skaggs School of Pharmacy and Pharmaceutical Sciences, University of California, San Diego, La Jolla, CA, United States, \\ ${ }^{2}$ Department of Neurosciences and Pharmacology, School of Medicine, University of California, San Diego, La Jolla, CA, \\ United States
}

The human tau protein is implicated in a wide range of neurodegenerative "tauopathy" diseases, consisting of Alzheimer's disease (AD) and frontotemporal lobar degeneration which includes progressive supranuclear palsy, corticobasal degeneration, Pick's disease, and FTLD-tau (frontotemporal dementia with parkinsonism caused by MAPT mutations). Tau gene transcripts in the human brain undergo alternative splicing to yield 6 different tau protein isoforms that are expressed in different ratios in neurodegeneration which result in tau pathology of paired-helical filaments, neurofibrillary tangles, and tau fibrillar aggregates with detrimental microtubule destabilization. Protease-mediated tau truncation is an important post-translational modification (PTM) which drives neurodegeneration in a tau fragment-dependent manner. While numerous tau fragments have been identified, knowledge of the proteolytic steps that convert each parent tau isoform into specific truncated tau fragments has not yet been fully defined. An improved understanding of the relationships between tau isoforms and their proteolytic processing to generate neurotoxic tau fragments is important to the field. This review evaluates tau isoform expression patterns including PTMs and mutations that influence proteolysis of tau to generate toxic fragments that drive cognitive deficits in AD and other tauopathy models. This assessment identifies the gap in the field on understanding the details of proteolytic steps used to convert each tau isoform into fragments. Knowledge of the processing mechanisms of tau isoforms can lead to new protease targeted drug strategies to prevent the formation of toxic tau fragments in tauopathy neurodegenerative diseases.

Keywords: tau isoforms, tau fragments, protease, mutations, neurotoxicity, tauopathies, Alzheimer's disease, frontotemporal dementia

\section{INTRODUCTION}

Accumulation of tau in neurofibrillary tangles (NFTs) and fibrillar aggregates participates in dementia and behavioral dysfunctions in numerous neurodegenerative diseases which include Alzheimer's disease $(\mathrm{AD})$ and frontotemporal lobar degeneration which includes progressive supranuclear palsy (PSP), corticobasal degeneration (CBD), Pick's disease (PiD), and FTLD-tau 
(frontotemporal dementia with parkinsonism caused by MAPT; Ballatore et al., 2007; Wolfe, 2012; Goedert et al., 2017). These brain disorders with tau pathogenesis are known as tauopathies. Human tau is a protein encoded by the microtubule associated protein tau (MAPT) gene on chromosome 17q21, which contains 14 exons (Martin et al., 2011; Caillet-Boudin et al., 2015; Goedert et al., 2017). Tau binds to microtubules (MT) and is essential for MT stabilization, assembly, and function in neuronal morphology and axonal transport (Weingarten et al., 1975; Cleveland et al., 1977; Ballatore et al., 2007). However, in tauopathies, tau loses its ability to bind to MTs through modifications of tau by phosphorylation (Alonso et al., 1994; Iqbal et al., 1994; Wang et al., 1995), proteolysis (Wang et al., 2007; Quinn et al., 2018), and other post-translational modifications (PTMs; Pevalova et al., 2006; Martin et al., 2011; Alquezar et al., 2020), as well as by tau mutations (Goedert and Spillantini, 2000, 2001; Spillantini and Goedert, 2000; Goedert, 2005; Goedert et al., 2017). The modified tau loses its MT stabilizing function and results in defective axonal transport (Kolarova et al., 2012; Combs et al., 2019). Modified tau dissociates from MTs and accumulates as NFTs or fibrillar aggregates resulting in disruption of cellular functions.

The molecular basis of aberrant tau forms that result in cellular dysfunctions, synaptic deficits, and cell death neurodegeneration has been an area of intense investigation. While mechanisms of tau phosphorylation have been studied extensively (Hanger et al., 2009; Wang et al., 2013; Šimić et al., 2016) less is known about the highly significant role of proteolysis of tau that generates numerous truncated fragments that drive neurodegeneration (Johnson, 2006; Quinn et al., 2018). The complete details of tau proteolysis to generate such fragments remains a gap in the field. For this reason, this review analyzes our current knowledge of the spectrum of tau fragments derived from multiple isoforms of tau to identify unanswered questions concerning the details of proteolytic steps utilized to convert each tau isoform into neurotoxic fragments. This assessment provides discussion of needed research in defining the spectrum of neurotoxic tau fragments specifically derived from each tau isoform by proteolysis. Complete knowledge of the proteolytic steps for producing neurotoxic tau fragments can lead to targeting of selected tau-cleaving proteases to inhibit production of highly toxic tau fragments in therapeutic strategies to alleviate tauopathy disease deficits.

\section{TAU ISOFORMS: IMBALANCE IN ISOFORM EXPRESSION PATTERNS IN TAUOPATHIES}

There are predominantly six different isoforms generated by alternative mRNA splicing mechanisms (Ballatore et al., 2007; Goedert et al., 2017) which range from 45 to $65 \mathrm{kDa}$ in length according to SDS gels (Liu and Gong, 2008; Figure 1A). Isoforms differ by the presence or absence of two 29 aa amino-terminal inserts encoded by exons 2 and 3 that are referred to as $0 \mathrm{~N}, 1 \mathrm{~N}$, or $2 \mathrm{~N}$ tau, and the presence or absence of the $\mathrm{R} 2$ domain, one of four partially repeated microtubule binding domain regions (MTBR) designated R1, R2, R3, and R4 (Figure 1A; Goedert et al., 1989, 2017; Barbier et al., 2019). Each R domain consists of four 3132 similar and distinct amino acid motifs (Figure 1B), encoded by exons 9-12 with exon 10 encoding R2. Isoforms expressing $\mathrm{R} 2$ are referred as $4 \mathrm{R}$ and those lacking R2 are referred as 3R (Goedert et al., 1989; Barbier et al., 2019). Altogether the six isoforms are named as $0 \mathrm{~N} 3 \mathrm{R}, 0 \mathrm{~N} 4 \mathrm{R}, 1 \mathrm{~N} 3 \mathrm{R}, 1 \mathrm{~N} 4 \mathrm{R}, 2 \mathrm{~N} 3 \mathrm{R}, 2 \mathrm{~N} 4 \mathrm{R}$, and are also referenced by the number of residues or clone name (Mandelkow and Mandelkow, 2012).

It is of interest that the ratio of $4 \mathrm{R}$ to $3 \mathrm{R}$ tau isoforms is approximately 1 in normal adult brain, but this ratio is differentially altered in neurodegenerative tauopathies, indicating an imbalance of $4 \mathrm{R}$ to $3 \mathrm{R}$ isoforms, regardless of whether the ratio shifts toward $4 \mathrm{R}$ or 3R (Gasparini et al., 2007). $\mathrm{AD}$ and frontotemporal dementia with parkinsonism, the nonAlzheimer's tauopathy, are classified as mixed $3 \mathrm{R}$ and $4 \mathrm{R}$ tauopathies (Hong et al., 1998). Other tauopathies such as $\mathrm{CBD}$ and $\mathrm{PiD}$ are characterized by their expression of specific tau isoforms. PiD is a tauopathy primarily affected by 3Rtau accumulation into "Pick bodies" (Buée and Delacourte, 1999; Yoshida, 2006). The molecular basis for altered expression profiles of tau isoforms in different tauopathies is unknown. Therefore, investigation of regulatory mechanisms that control the biosynthesis of tau isoforms through tau gene transcription, RNA alternative splicing, and protein translation will advance understanding of the molecular basis for differential expression of tau isoforms among the different tauopathies.

Tau isoforms display differences in aggregation properties. Rates of assembly between $4 \mathrm{R}$ and $3 \mathrm{R}$ tau isoforms are distinct, with $4 \mathrm{R}$ tau isoforms assembling 2.5-3.0 times more rapidly compared to $3 \mathrm{R}$ tau isoforms, with no contributions by differences in amino-terminal insertions (Goedert and Jakes, 1990). These differences in assembly of $4 \mathrm{R}$ compared to $3 \mathrm{R}$ tau isoforms indicate variations in the kinetics of aggregation of $4 \mathrm{R}$ compared to $3 \mathrm{R}$ tau isoforms. Differences in aggregation properties of tau isoforms implicate heterogeneity in the compositions of tau aggregates among tauopathies expressing altered ratios of $4 \mathrm{R}$ and $3 \mathrm{R}$ tau isoforms.

Furthermore, tau isoform expression patterns and phosphorylation are developmentally regulated. The smallest tau isoform $(0 \mathrm{~N} 3 \mathrm{R})$ is the only form that is expressed in fetal human brain and is highly phosphorylated compared to adult tau (Goedert et al., 1993; Brion et al., 1994). A switch from $3 \mathrm{R}$ to $4 \mathrm{R}$ tau in mouse brain occurs between postnatal day 9 (P9) to P18 under the same time course as a conversion from high to low phosphorylation (Tuerde et al., 2018). It is noted that highly phosphorylated fetal tau is functional and does not polymerize into NFTs or fibrillar aggregates (Yu et al., 2009). This group compared individual phosphorylation sites of tau in the developing rat brain and pathological tau from human AD brain. It was found that phosphorylations at S202, T212, T231, S396, S404, and S422 were much higher in AD brain than in developing rat brain, whereas the phosphorylation levels of T181, S199, T205, S214, S262, S356, and S409 in AD brain were similar or lower than tau in the developing rat brain. This data suggests that different phosphorylation levels at selective sites of the tau 


\section{A CNS Tau}

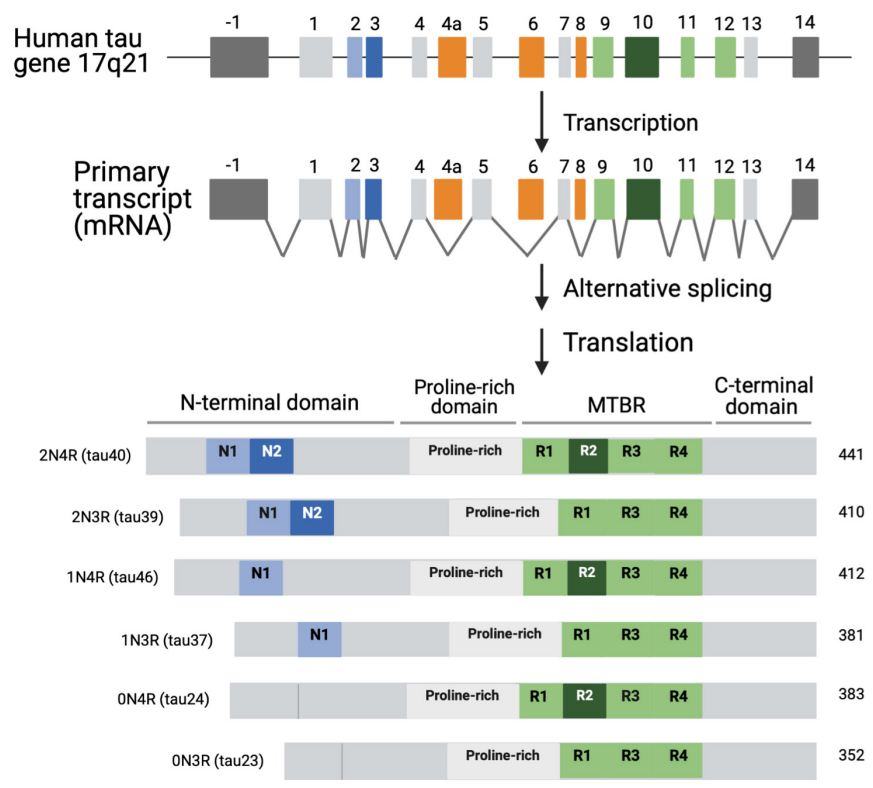

B

\section{Sequences of R1-R4 domains}

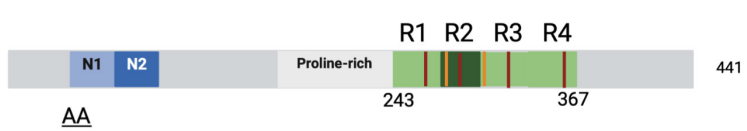

243 LQTAPVPMPDLKNVKSKIGSTENLKHQPGGG R1

274 KVQIINKKLDLSNVQSKCGSKDNIKHVPGGG R2

305 SVQIVYKPVDLSKVTSKCGSLGNIHHKPGGG R3

336 QVEVKSEKLDFKDRVQSKIGSLDNITHVPGGG R4

FIGURE 1 | Tau isoforms generated through tau gene expression, alternative RNA splicing, and protein translation. (A) Tau biosynthesis in the CNS. Tau is found on chromosome 17 arm 21. The tau gene contains 16 exons. Exons 1, 4, 5, 7, 13 (light gray) and 9, 11, 12 (light green) are all constitutively transcribed in the CNS (Martin et al., 2011). Exons 9, 11, and 12 encode the R1, R3, and R4 domains, respectively (Martin et al., 2011; Goedert et al., 2017 ). Exon 10 encodes the R2 domain. Exon -1 is part of the promotor region and is not translated. Exons 4A, 6, and 8 (orange) are mostly expressed in peripheral tissues. Exon 14 is a part of the 3' untranslated region of the mRNA sequence and is not translated (Goedert et al., 1989; Andreadis, 2005). Tau isoforms are generated by alternate splicing of exons 2 and 3 (light and dark blue) and exon 10 (dark green). The six isoforms range from 352 to 441 amino acids and can be referred to by the clone name as well. Structurally, tau is subdivided into the N-terminal domain, the proline rich domain, microtubule-binding domain region, and the $\mathrm{C}$-terminal domain. (B) $\mathrm{R}$ domain MTBR primary sequences. The primary amino acid sequences of the microtubule binding region (MTBR) shows that R1 (243-273), R2 (274-304), R3 (305-335), and R4 (336-367) are partially repeated sequences (Barbier et al., 2019). 275 VQIINK 280 and 306 VQIVYK 311 are two motifs within R2 and R3 that have strong MT interactions and are important for intermolecular $\beta$-sheet formation (Moreno-Castillo et al., 2020).

protein may play a key role in converting tau from a biologically active molecule into one that inhibits MT assembly.

Studies investigating the regulation of tau phosphorylation have also been studied in human fetal and adult autopsy brain tissue (Hefti et al., 2019). Of the 20 fetal cases that showed immunoreactivity to at least one of the tau antibodies used, $18 / 20$ (90\%) showed positive staining for phosphorylation at S214 and 17/20 (85\%) showed positive staining for PHF1 antibody (pS396, pS404). Only 4 cases were positive for AT8 staining of pS202 and pT205, and 2 for RZ3 staining of pT231. Four serine residues (S214, S396, S404, and S202) appeared to be phosphorylated in both fetal and $\mathrm{AD}$ cases. Interestingly, the investigators (Hefti et al., 2019) found phospho-tau positive aggregates in fetal human brain. These aggregates were positive to T214 and weakly immunoreactive to CP3 antibody (S202) and PHF-1. The aggregates appeared to be non-toxic as they were negative for thioflavin $S$ staining, indicating that they were seed-incompetent.

It is not fully clear how the transition of the isoforms and phosphorylation are regulated, and further investigation is still needed. Moreover, it will be important to gain understanding of the propensity of distinct tau isoforms to become phosphorylated, the hallmark of neurodegeneration in tauopathies that occur in aging.

\section{TAU PATHOLOGY INVOLVING HYPERPHOSPHORYLATION}

Tau pathology manifests as the deposition of insoluble aggregated NFTs, fibrillar aggregates, neuropil threads, paired helical fragments (PHFs), and straight filaments (ST) resulting from hyperphosphorylation of normal tau which leads to decreased tau affinity to MT (Figure 2; Wischik et al., 1988; Perry et al., 1991; Wille et al., 1992; Fitzpatrick et al., 2017). Upon self-assembly into PHF and STs, abnormally hyperphosphorylated cytosolic tau loses its ability to sequester normal microtubule associated proteins and disrupts microtubule assembly (Ballatore et al., 2007; Iqbal et al., 2010). Thus, it is a combination of loss-of-regular function consisting of detachment from MTs and loss of MT stabilization, as well as a toxic gain-of-function of PHF and NFT formation with derangement of normal cellular transport that contribute to tau pathology.

Interestingly, tau aggregates from $\mathrm{AD}$ brain compared to those from chronic traumatic encephalopathy display structural differences (Fitzpatrick et al., 2017; Falcon et al., 2019; Shi et al., 2021). Such findings suggest possible heterogeneity of tau aggregates and composition of tau fragments among different neurodegenerative disease conditions. 


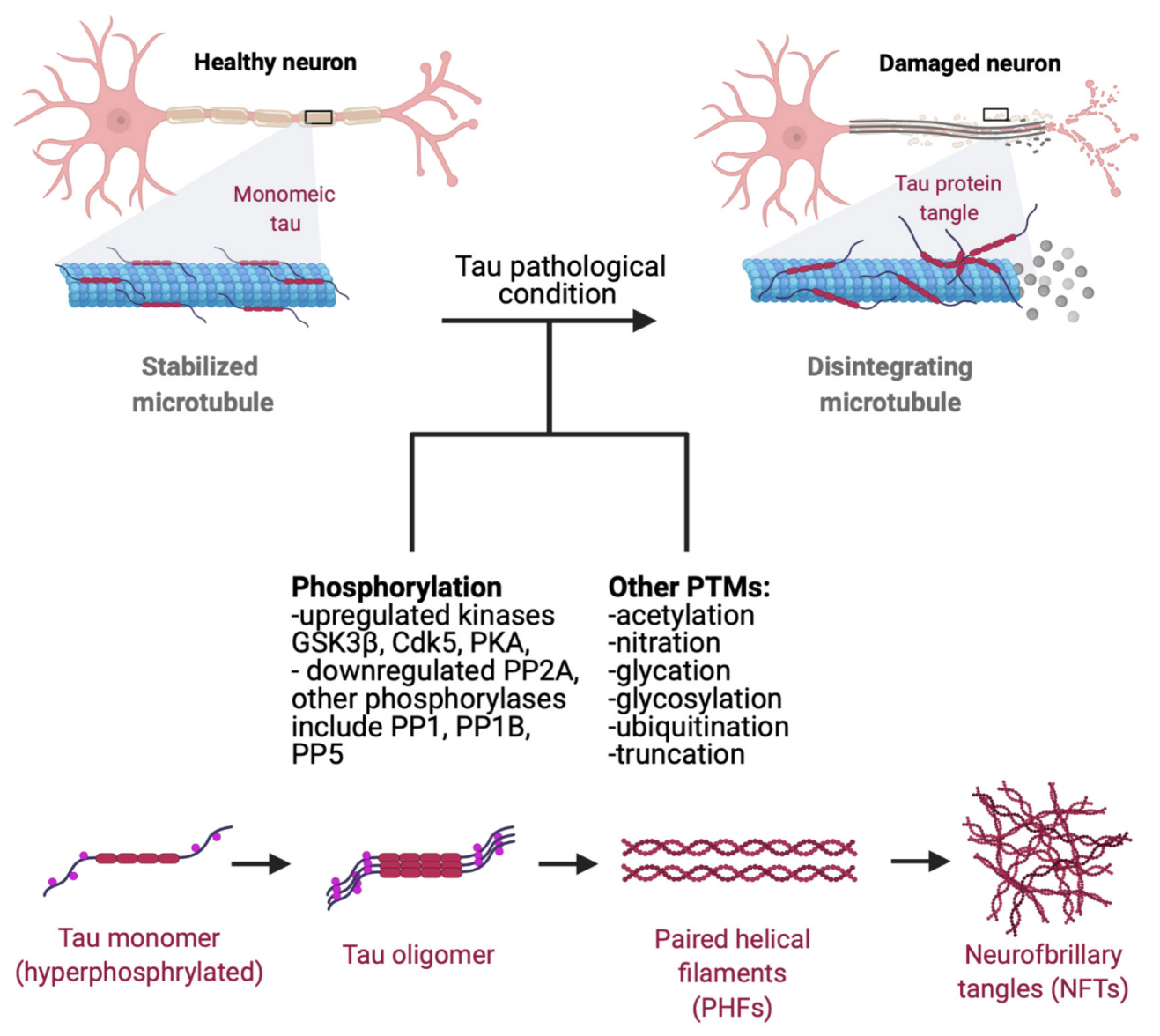

FIGURE 2 | Progression of tau pathology. Microtubules are stabilized by monomeric tau through the MTBR (microtubule binding region). Hyperphosphorylation decreases tau stability and causes p-tau to dissociate from microtubules, leading to disintegration and disruption of normal axonal transport. Hyperphosphorylated tau gains affinity for other tau monomers, forming oligomeric species, adapting a $\beta$-sheet structure prior to NFT and aggregate formation (Maeda et al., 2006). Dissociated tau has a tendency to dimerize in an antiparallel fashion, self-assembling into dimers (Wille et al., 1992). These manifest later into PHFs and ultimately NFTs and fibrillar aggregates which can propagate and mediate cytotoxicity in neuronal cells. Phosphorylation of tau by GSK3 $\beta$ and Cdk5 affects tau-microtubule interactions by reducing tau's microtubule affinity (Wagner et al., 1996), with protein kinase A (PKA) phosphorylation at S262 and S214 demonstrating similar effects (Biernat et al., 1993; Schneider et al., 1999). Recent studies have also shown that protein phosphatases (PP) PP1, PP2A, PP2B, and PP5 all dephosphorylate tau in vitro, with PP2A functioning as a major regulator of phosphorylation at multiple sites in the brain. PP2A is also partially down-regulated in AD brains in response to tubulin assembly (Sontag et al., 1999; Liu et al., 2005). Tau also goes a plethora of post-translational modifications such as acetylation, nitration, glycation, glycosylation, ubiquitination, and truncation, many of which are known to increase tau's propensity to aggregate (Alquezar et al., 2020).

Significant spreading of tau pathology occurs in brain (Fuster-Matanzo et al., 2018; Franzmeier et al., 2020) and may involve tau secretion (Pernègre et al., 2019; Merezhko et al., 2020). Tau pathology and its propagation has been investigated in animal models of tauopathies. In transgenic mouse models overexpressing human tau P301L restricted to the entorhinal cortex (EC-II), tau pathology propagated through synaptic circuits to the dentate gyrus, CA fields of the hippocampus, and the cingulate cortex (de Calignon et al., 2012). P-tau aggregates also propagate to the frontal and temporal cortices as well as surrounding isocortex areas (Hu et al., 2017). Together, this data supports circuitbased, neuron-to-neuron tau transmission and correlates with the behavioral symptoms of $\mathrm{AD}$ including memory and cognitive deficits.

\section{OTHER POST TRANSLATIONAL MODIFICATIONS IN THE CONTEXT OF TAU PATHOLOGY}

Tau is a natively unfolded and intrinsically disordered protein that is remarkably resistant to hydrophobicity-driven collapse due to the lack of hydrophobic residues and is highly susceptible to aggregate within a wide range of $\mathrm{pH}$ values between 5 and 10 (Jeganathan et al., 2008). As such, in addition to phosphorylation, tau is especially prone to other forms of PTMs including (but not limited to) acetylation, ubiquitination, SUMOylation, glycation, and glycosylation (Park et al., 2018; Alquezar et al., 2020). It is of interest to understand the relationship of PTMs with tau phosphorylation in the regulation of toxic tau fragments and aggregation pathology. 
Acetylation of mammalian proteins involves the N-terminal addition of acetyl groups from acetyl coenzyme A to lysine residues of polypeptide chains (Drazic et al., 2016). Acetylation of tau is enhanced in patients at early/moderate Braak states (Min et al., 2010). Disease and context specific abnormalities of HATs (histone acetyltransferase) and HDACs (histone deacetylases) involved in tau acetylation have been observed (Min et al., 2015), but these abnormalities are both context and disease specific. For example, p300/CBP HAT activity is increased in frontotemporal lobar degeneration patients (Chen et al., 2020), but lowered in the F2 area of the frontal cortex and hippocampus of AD patients compared to aged controls (Schueller et al., 2020). Tau acetylation at $\mathrm{K} 174, \mathrm{~K} 274$, and $\mathrm{K} 281$ has been shown to promote cognitive and synaptic defects and impaired hippocampal longterm potentiation (Min et al., 2015; Tracy and Gan, 2017). K280 acetylation has distinct pathological signature markings in different tauopathies and was absent in control brain tissue or cultured wild-type neurons, showcasing the disease-specific nature of K280 acetylation (Irwin et al., 2012). Interestingly, tau isoforms have different propensities to undergo lysine acetylation, with auto-acetylation occurring more prominently in the 2nd and 4th lysine-rich MTBRs, which induced proteolytic tau cleavage and generated distinct $\mathrm{N}$ - and C-terminal fragments (Cohen et al., 2016). This group found that auto-acetylation of full-length tau 0N4R leads to production of distinct tau fragments of $43,38,17$, and $12 \mathrm{kDa}$ tau fragments, a significant reduction in full-length protein, and an approximately 10-fold difference in tau-K18 fragmentation.

Ubiquitination is another covalent modification of proteins on lysine residues that targets proteins for degradation by the $26 \mathrm{~S}$ proteasome (Stringer and Piper, 2011) and the autophagylysosome pathway (Mukhopadhyay and Riezman, 2007; Nathan et al., 2013). It was found that 17 out of a total of 44 lysine residues of tau were ubiquitinated, with the majority found in the MTBR (Munari et al., 2020). Ubiquitin has been found in aggregated tau extracted from tauopathy human brain tissues (Bancher et al., 1991; García-Sierra et al., 2012). Strong association has been found between ubiquitin and an early truncation event at D421 (García-Sierra et al., 2012). These findings suggest interrelationships between phosphorylation and ubiquitination of tau in its aggregated pathology.

SUMOylation is the transfer of a ubiquitin-like protein SUMO to the terminal amino group of lysine side chains and plays roles in a wide range of neurodegenerative diseases (Henley et al., 2014; Anderson et al., 2017). In particular, monoSUMOylation in vitro occurs at the $\mathrm{K} 340$ in R4 and inhibits tau ubiquitination and subsequent proteasome-dependent degradation, potentially inducing tau accumulation and aggregation (Luo et al., 2014). It was found that $70-75 \%$ of lysosomes in inclusion body-positive oligodendrocytes of patients with multiple system atrophy and PSP were SUMO-1 positive with tau, suggesting a role in autophagy-lysosome pathways (Wong et al., 2013).

Glycation (or non-enzymatic glycosylation) is a PTM in which sugars or sugar-derived metabolites are covalently attached to side chains of lysine residues (Li et al., 2012; Rungratanawanich et al., 2021). Glycation processes also involve a set of heterogenous modifications that lead to advanced glycation end-products, which are irreversible cross-links between glycated and non-glycated proteins with relevance to age-related diseases (Sasaki et al., 1998; Simm et al., 2015). Thirty-two lysine residues of tau have been identified to be glycated in vitro (Nacharaju et al., 1997; Liu et al., 2016). Many sites are detected in both $3 \mathrm{R}$ and $4 \mathrm{R}$, but the presence of K280 and K281 in only 4R isoforms indicate that $4 \mathrm{R}$ isoforms (and specifically $2 \mathrm{~N} 4 \mathrm{R}$ which has the highest number of $\mathrm{K}$ residues) with glycation may be related to the higher tendency of $4 \mathrm{R}$ isoforms to aggregate.

O-glycosylation is an example of a PTM that has been hypothesized to protect tau from phosphorylation (Liu et al., 2004). O-GlyNAcylation modification of tau inhibits its aggregation (Yuzwa et al., 2012, 2014) and modulates the formation of tau paired helical filaments (Arnold et al., 1996).

While there is evidence for extensive tau PTMs of differing types, the specific roles of these modifications on proteolytic processing of tau is not well known. It will be important in future studies to assess how PTMs may influence tau proteolysis in generating neurotoxic tau fragments.

\section{TAU MUTATIONS AFFECT ISOFORM RATIOS}

Over 50 different pathogenic MAPT missense, silent, and intronic mutations have been reported to be present in different sporadic tauopathies such as PSP, CBD, PiD, and FTLD-tau (frontotemporal dementia with parkinsonism caused by MAPT mutations; D’Souza et al., 1999; D’Souza and Schellenberg, 2005; Goedert et al., 2017; Strang et al., 2019). The majority of tau mutations are clustered in the microtubule binding repeat domains of tau (Figure 3). The mutations are composed of two main classes.

The first class includes missense and deletion changes in the coding region and induces the ability of tau to aggregate and form filaments (Arrasate et al., 1999; Goedert et al., 1999; D'Souza and Schellenberg, 2005). This class of mutations includes G272V, $\Delta 280 \mathrm{~K}, \mathrm{P} 301 \mathrm{~L}, \mathrm{~V} 337 \mathrm{M}, \mathrm{R} 406 \mathrm{~W}$ which have all been found in FTLD-tau patients and caused moderate decreases in microtubule interaction and stabilization (Barghorn et al., 2000; D'Souza and Schellenberg, 2005). P301L particularly increases aggregation potential and largely consists of mutant 4R-tau, with only small amounts of normal 4R- and 3R-tau (Izzu et al., 2000; D'Souza and Schellenberg, 2005).

The second class of mutations affect alternate slicing of MAPT transcripts. These mutations mostly affect exon 10 splicing, and include N279K, L284L, S305N, S305S, $\Delta 296 \mathrm{~N}$, and N296H (Goedert et al., 2000; Miyamoto et al., 2001). These specific mutations alter exon 10 splicing and result in an increase in the ratio of $4 \mathrm{R}$ - to $3 \mathrm{R}$-tau, indicating that $4 \mathrm{R}$-tau is more toxic (Hutton et al., 1998). In an examination of autopsy material available from mutation carriers, there was an increase in the 4R/3R ratio (Spillantini et al., 1998; Goedert et al., 1999). While it remains unclear fully as to how this ratio leads to aberrant microtubule function, several hypotheses are being examined. One proposed mechanism is that $4 \mathrm{R}$ - tau binds to MTs with 


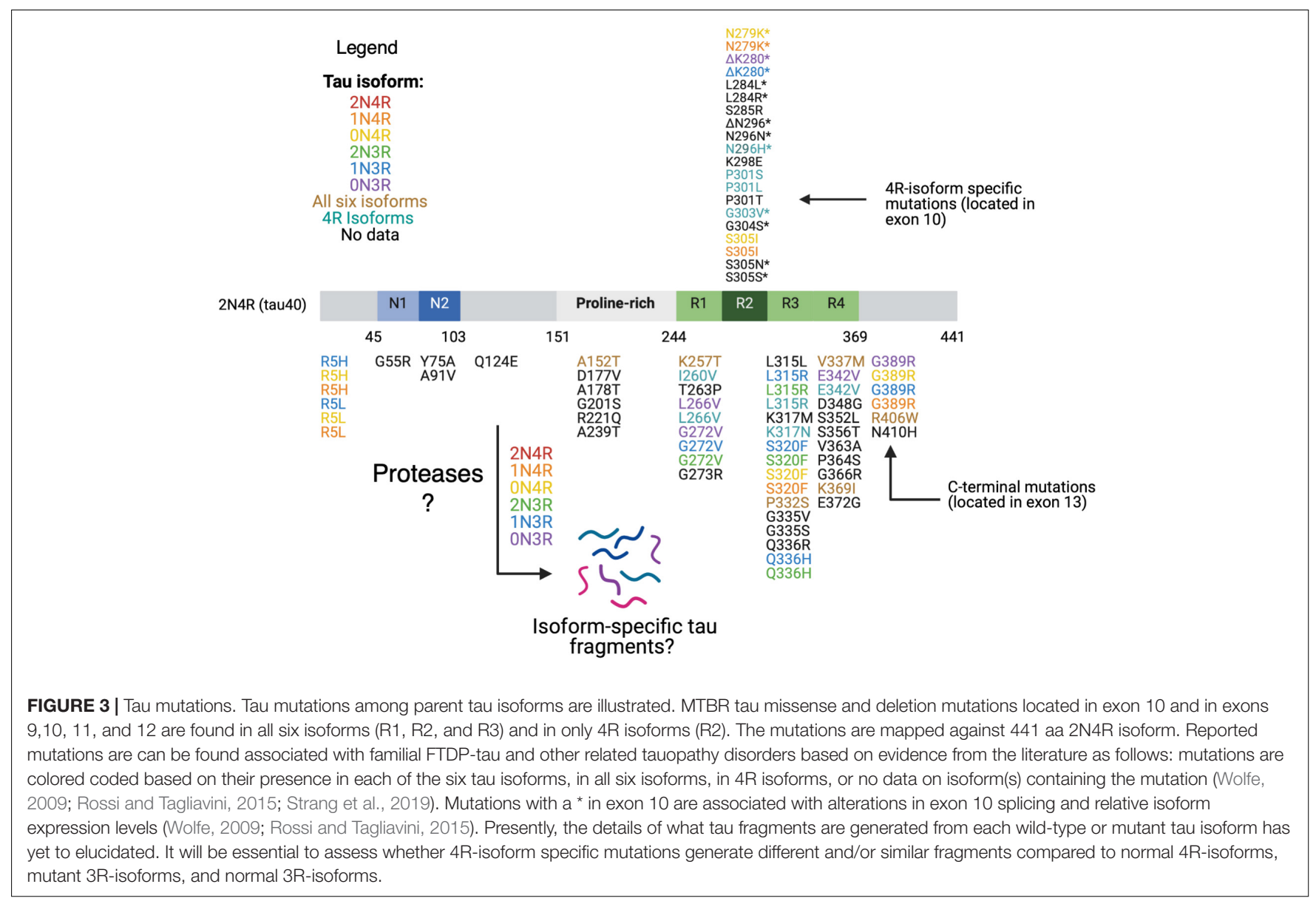

higher affinity due to its extra microtubule binding domain and displaces 3R-tau, resulting in excess free 3R-tau (Hong et al., 1998; D'Souza and Schellenberg, 2005). Alternative proposals suggest there are discrete MT binding sites for $3 \mathrm{R}$ and $4 \mathrm{R}$ tau (Goode et al., 2000; Makrides et al., 2004), and the synthesis of excess $4 \mathrm{R}$ tau results in saturation of the $4 \mathrm{R}$ sites, causing an excess of free $4 \mathrm{R}$ tau, which can aggregate (D'Souza and Schellenberg, 2005). This has been shown to occur in cell culture experiments overexpressing $4 \mathrm{R}$ tau ( $\mathrm{Lu}$ and Kosik, 2001). One mutation at position $280, \Delta 280 \mathrm{~K}$, is unique in that it involves deletion of a lysine (mutation at exon 10) and reduces the $4 \mathrm{R} / 3 \mathrm{R}$ ratio via ablation of exon 10 splicing (Van Swieten et al., 2007; Momeni et al., 2009).

\section{TAU FRAGMENTS DRIVE MEMORY DEFICITS AND TOXICITY IN TRANSGENIC ANIMAL MODELS}

Tau proteolytic fragments have been shown to drive memory deficits and toxicity in tau animal models of neurodegenerative tauopathies. Numerous tau fragments induce tau pathology and behavioral deficits in in vivo animal models of tauopathies (Table 1), and induce in vitro cellular toxicities (Table 2).
In rTg4510 mice expressing human mutant tauP301L, levels of the $35 \mathrm{kDa}$ tau fragment, also known as $\Delta$ tau314, correlates with memory deficits (Zhao et al., 2016). The rTg4510 mice expressed transgenic tauP301L driven by a $\mathrm{Ca}^{2+} /$ calmodulin kinase II promoter system in the forebrain (Ramsden et al., 2005). Memory deficits are observed between 2 and 3 months of age, several months before the loss of synapses or neurons occurring at 4 to 9 months (Ramsden et al., 2005; Santacruz et al., 2005). Blockade of the formation of $\Delta$ tau314 by mutagenesis of the caspase- 2 cleavage site at $\mathrm{D} 314 \mathrm{E}$ resulted in improved memory deficits, which supports involvement of $\Delta$ tau314 in the behavioral deficit (Zhao et al., 2016). Fluorescence and sedimentation assays compared fibrillation of $\Delta$ tau314, full-length, and tauP301; $\Delta$ tau314 showed the lowest propensity to form fibrils, suggesting that there is a different mechanism by which it impairs brain function.

A study of another $35 \mathrm{kDa}$ C-terminal tau fragment was conducted without mutations by expression in Tau35 transgenic mice, and results showed that this $35 \mathrm{kDa}$ tau fragment is sufficient to result in cognitive deficits (Bondulich et al., 2016). Tau35 mice express the C-terminal half of wild-type human tau (aa 187-441) fused at the C-terminus of the haemagglutinin tag and under control of a human tau promoter and targeted to a Hprt locus. Transgene expression in Tau35 mice is only 
TABLE 1 | Tau fragments in in vivo models displaying memory deficits and/or cellular toxicity.

\begin{tabular}{|c|c|c|c|c|c|}
\hline Tau fragment & $\begin{array}{l}\text { Description of } \\
\text { transgenic }(\mathrm{Tg}) \\
\text { mouse, and } \\
\text { transgenes }\end{array}$ & $\begin{array}{l}\text { Memory and } \\
\text { behavioral deficits: } \\
\text { observed, none, or } \\
\text { unknown }\end{array}$ & $\begin{array}{l}\text { Toxicity mechanisms: } \\
\text { observed, none, or } \\
\text { unknown }\end{array}$ & $\begin{array}{l}\text { Associated } \\
\text { proteases }\end{array}$ & References \\
\hline $\begin{array}{l}1-314 \\
(\Delta \text { tau314, } 35 \\
k D a)\end{array}$ & $\begin{array}{l}\text { rTg4510 mice } \\
\text { expressing tau }{ }^{P 01 L} \text {, } \\
\text { TCP35 tau fragment } \\
\text { (35 kDa), also known } \\
\text { as } \Delta \text { tau314, compared } \\
\text { to wild-type (wt) tau }\end{array}$ & $\begin{array}{l}\text { Memory deficit } \\
\text { observed and } \\
\text { correlated with levels of } \\
\text { TCP35 tau fragment } \\
\text { ( } \Delta \text { tau314) }\end{array}$ & $\begin{array}{l}\text { Impaired synaptic } \\
\text { transmission. glutamate } \\
\text { receptor dislocation, } \\
\text { hippocampal neuronal } \\
\text { loss }\end{array}$ & $\begin{array}{l}\text { Caspase-2 cleaves at } \\
\text { D314-L315 (Zhao et al., } \\
\text { 2016) }\end{array}$ & Zhao et al., 2016 \\
\hline $\begin{array}{l}187-441 \\
\text { (C-terminal } 35 \\
\text { kDa fragment) }\end{array}$ & $\begin{array}{l}\text { Tau35 mice model } \\
\text { expressing a } 35 \mathrm{kDa} \\
\text { tau fragment without } \\
\text { mutations and under } \\
\text { control of the human } \\
\text { tau promotor }\end{array}$ & $\begin{array}{l}\text { Memory deficit and } \\
\text { motor dysfunction } \\
\text { observed }\end{array}$ & $\begin{array}{l}\text { Increased tau } \\
\text { phosphorylation, } \\
\text { activation of GSK3b } \\
\text { kinase, compromised } \\
\text { synaptic function, and } \\
\text { impairment of } \\
\text { autophagy and } \\
\text { lysosome- mediated } \\
\text { degradation }\end{array}$ & $\begin{array}{l}\text { Unknown at } \\
\text { G186-E187 }\end{array}$ & Bondulich et al., 2016 \\
\hline $\begin{array}{l}1-255 \text { and } \\
1-368\end{array}$ & $\begin{array}{l}\text { Tg mice expressing } \\
\text { P301S display tau } \\
\text { fragments N255 and } \\
\text { N368, generated by } \\
\text { AEP. }\end{array}$ & $\begin{array}{l}\text { Memory deficit } \\
\text { observed }\end{array}$ & $\begin{array}{l}\text { AEP is up-regulated, } \\
\text { and responsible for } \\
\text { synapse loss and } \\
\text { cognitive deficits shown } \\
\text { by AEP knockout. }\end{array}$ & $\begin{array}{l}\text { AEP cleaves at } \\
\text { N255-V256 and } \\
\text { N368-K369 }\end{array}$ & Zhang et al., 2014 \\
\hline $151-421(\Delta \mathrm{tau})$ & $\begin{array}{l}\text { TAU62 Tg mice } \\
\text { co-expressing 3R tau } \\
\text { 151-421 ( } \Delta \text { tau) } \\
\text { crossed with a } 383 \text { aa } \\
\text { 4R tau with a P301S } \\
\text { mutation } \\
\text { (P301SxTAU62), Also } \\
\text { studied TAU62 mice } \\
\text { crossed with ALZ17 Tg } \\
\text { mice expressing } \\
\text { wild-type 4R tau } \\
\text { AlZ717xTAU62 }\end{array}$ & $\begin{array}{l}\text { Memory deficit and } \\
\text { motor abnormalities } \\
\text { observed in all crossed } \\
\text { mice. These included } \\
\text { gait ataxia, tremor, and } \\
\text { hindlimb reflex deficits }\end{array}$ & $\begin{array}{l}\text { Disruption of axonal } \\
\text { transport, } \\
\text { mitochondria, Golgi } \\
\text { apparatus, and } \\
\text { synaptic proteins; co- } \\
\text { expression led to } \\
\text { severe paralysis within } \\
3 \text { weeks and was } \\
\text { rescued by cessation of } \\
\text { tau } 151-421 \text { expression }\end{array}$ & $\begin{array}{l}\text { ADAM-10 cleaves } \\
\text { nearby at 1151-A152 } \\
\text { (Henriksen et al., 2013), } \\
\text { Caspase-2 cleaves at } \\
\text { 0421-S422 (Zhao et al., } \\
\text { 2016) }\end{array}$ & Ozcelik et al., 2016 \\
\hline $\begin{array}{l}\text { 45-230 (17 } \\
\mathrm{kDa})\end{array}$ & $\begin{array}{l}\text { Tg mice expressing } \\
17 \mathrm{kDa} \text { tau } 45-230 \\
\text { compared to wt mice }\end{array}$ & $\begin{array}{l}\text { Memory and learning } \\
\text { abnormalities } \\
\text { observed, enhanced } \\
\text { fear response (fear } \\
\text { conditioning test) and } \\
\text { longer time in the } \\
\text { morris water maze test. }\end{array}$ & $\begin{array}{l}\text { Tau } 45-230 \text { expression } \\
\text { resulted in synaptic loss } \\
\text { and alterations in } \\
\text { NMDA receptor } \\
\text { subunits }\end{array}$ & $\begin{array}{l}\text { Calpain-1 cleaves at } \\
\text { K44-E45 (Yang and } \\
\text { Ksiezak-Reding, 1995), } \\
\text { Calpain 1- and 2- } \\
\text { cleave at R230-T231 } \\
\text { (Garg et al., 2010) }\end{array}$ & Lang et al., 2014 \\
\hline $\begin{array}{l}\text { 243-441 } \\
\text { (Tau-CTF24, } 24 \\
\text { kDa) }\end{array}$ & $\begin{array}{l}\text { Tg601 mouse } \\
\text { overexpressing } \\
\text { wild-type human tau } \\
\text { (2N4R) that developed } \\
\text { hyperphosphorylated- } \\
\text { tau }\end{array}$ & $\begin{array}{l}\text { Memory deficit } \\
\text { observed }\end{array}$ & $\begin{array}{l}\text { Accelerated intracellular } \\
\text { propagation of tau, } \\
\text { reduced capacity for } \\
\text { MT assembly } \\
\text { compared to tau441 }\end{array}$ & $\begin{array}{l}\text { Calpain } 1 \text { cleaves at } \\
\text { R242-L243 } \\
\text { (Matsumoto et al., } \\
\text { 2015) }\end{array}$ & $\begin{array}{l}\text { Park and Ferreira, 2005; Garg } \\
\text { et al., 2010; Matsumoto et al., } \\
2015\end{array}$ \\
\hline
\end{tabular}

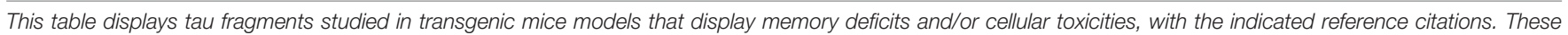
studies demonstrate that transgenic mice models are useful to the field to study behavioral and memorial abnormalities involving tau fragments.

approximately $7 \%$ of the total amount of tau and thus nonphysiological functions of over-expressed tau were avoided. Importantly, the $35 \mathrm{kDa}$ tau fragment is present in human tauopathies and provides a model for disorders such as $\mathrm{AD}$, PSP, and CBD. In the study, Tau35 mice exhibited age-related limb clasping with an incidence rate of $5 \%$ by 4 months, an increase to $25 \%$ by $5-6$ months, and all mice showing paresis by 18 months. At 4 months, Tau35 mice exhibited a kyphosis index (KI) of 4.5 , which decreased to 3.4 at 8 months and 2.9 by
14 months, indicating a reduction in $\mathrm{KI}$ demonstrates progressive age-related kyphosis. These mice also exhibited molecular deficits such as increased tau phosphorylation, activation of GSK $\beta$, and impairment in autophagy and lysosomal-mediated degradation.

Another transgenic mice model looked at the role of arginine endopeptidase AEP (Lmnn gene) in synaptic function and behavior by crossing Lgmn $^{-/-}$mice with P301S-transgenic mice to knock out AEP (Zhang et al., 2014). At 6 months of age, tau P301S-transgenic mice displayed overt reductions in synapses 
TABLE 2 | Tau fragments in in vitro models displaying cellular toxicity.

\begin{tabular}{|c|c|c|c|c|}
\hline $\begin{array}{l}\text { Tau } \\
\text { fragment }\end{array}$ & $\begin{array}{l}\text { Description } \\
\text { of in vitro } \\
\text { model }\end{array}$ & $\begin{array}{c}\text { Toxicity } \\
\text { mechanisms } \\
\text { of tau }\end{array}$ & $\begin{array}{l}\text { Associated } \\
\text { protease }\end{array}$ & References \\
\hline $1-44$ & $\begin{array}{c}\text { Cerebellar } \\
\text { granule cells } \\
\text { from 8-day } \\
\text { rats or from } \\
6 \text {-day-old } \\
\text { ERK1-/- } \\
\text { mice }\end{array}$ & $\begin{array}{l}\text { Cell death } \\
\text { involving } \\
\text { extrasynaptic } \\
\text { NMDAR } \\
\text { excitotoxicity } \\
\text { + ERK1/2 } \\
\text { pathway }\end{array}$ & $\begin{array}{l}\text { Calpain-1 } \\
\text { cleaves at } \\
\text { K44-E45 }\end{array}$ & $\begin{array}{l}\text { Amadoro et al., } \\
\text { 2006; Yang and } \\
\text { Ksiezak- } \\
\text { Reding, } \\
1995\end{array}$ \\
\hline $26-44$ & $\begin{array}{l}\text { Cerebellar } \\
\text { granule cells } \\
\text { from 8-day } \\
\text { rats or from } \\
\text { 6-day-old } \\
\text { ERK1-/- } \\
\text { mice }\end{array}$ & $\begin{array}{l}\text { Cell death } \\
\text { involving } \\
\text { extrasynaptic } \\
\text { NMDAR } \\
\text { excitotoxicity } \\
\text { + ERK1/2 } \\
\text { pathway }\end{array}$ & $\begin{array}{l}\text { Caspase-3 } \\
\text { cleaves at } \\
\text { K25-Q26, } \\
\text { Calpain-1 } \\
\text { cleaves at } \\
\text { K44-E45 }\end{array}$ & $\begin{array}{c}\text { Amadoro et al., } \\
\text { 2006; Yang and } \\
\text { Ksiezak- } \\
\text { Reding, } \\
1995\end{array}$ \\
\hline $26-230$ & $\begin{array}{c}\text { Adenoviral } \\
\text { expression of } \\
\text { tau } 26-230 \text { in } \\
\text { primary } \\
\text { neuronal cells, } \\
\text { and } \\
\text { differentiated } \\
\text { neuroblastoma } \\
\text { cells } \\
\text { undergoing } \\
\text { apoptosis by } \\
\text { BDNF } \\
\text { withdrawal or } \\
\text { treatment with } \\
\text { staurosporine }\end{array}$ & $\begin{array}{l}\text { NMDA- } \\
\text { mediated } \\
\text { neurotoxicity }\end{array}$ & $\begin{array}{l}\text { Caspase-3 } \\
\text { cleaves at } \\
\text { 025-026, } \\
\text { Calpain-1 and } \\
\text {-2 cleave at } \\
\text { R230-T231 }\end{array}$ & $\begin{array}{c}\text { Corsetti et al., } \\
2008\end{array}$ \\
\hline $1-156$ & $\begin{array}{c}\text { Cerebellar } \\
\text { granule cells } \\
\text { obtained from } \\
\text { 8-day rats or } \\
\text { from } \\
\text { 6-day-old } \\
\text { ERK1/mice }\end{array}$ & Unknown & $\begin{array}{c}\text { Thrombin } \\
\text { cleaves nearby } \\
\text { site at } \\
\text { R155-G156 }\end{array}$ & $\begin{array}{c}\text { Amadoro et al., } \\
\text { 2006; Arai } \\
\text { et al., } 2005\end{array}$ \\
\hline $1-255$ & $\begin{array}{c}\text { Primary } \\
\text { neurons } \\
\text { transfected } \\
\text { with adeno- } \\
\text { associated } \\
\text { viruses } \\
\text { encoding tau } \\
\text { 1-255 }\end{array}$ & $\begin{array}{c}\text { Tau } \\
\text { aggregation } \\
\text { into PHF with a } \\
\text { mixture of tau } \\
\text { fragments }\end{array}$ & $\begin{array}{l}\text { AEP cleaves at } \\
\text { N255-V256 }\end{array}$ & $\begin{array}{l}\text { Zhang et al., } \\
2014\end{array}$ \\
\hline $1-368$ & $\begin{array}{c}\text { Primary } \\
\text { neurons } \\
\text { transfected } \\
\text { with adeno- } \\
\text { associated } \\
\text { viruses } \\
\text { encoding tau } \\
1-368\end{array}$ & $\begin{array}{l}\text { Tau assembly } \\
\text { into filamentous } \\
\text { structures and } \\
\text { PHF, triggered } \\
\text { apoptosis }\end{array}$ & $\begin{array}{c}\text { AEP cleaves at } \\
\text { N368-K369 }\end{array}$ & $\begin{array}{c}\text { Zhang et al., } \\
2014\end{array}$ \\
\hline 256-368 & $\begin{array}{c}\text { Primary } \\
\text { neurons } \\
\text { transfected } \\
\text { with adeno- } \\
\text { associated } \\
\text { viruses } \\
\text { encoding tau } \\
256-268\end{array}$ & $\begin{array}{c}\text { Tau assembly } \\
\text { into filamentous } \\
\text { structures and } \\
\text { PHF, triggered } \\
\text { apoptosis }\end{array}$ & $\begin{array}{l}\text { AEP cleaves at } \\
\text { N255-V256 } \\
\text { and } \\
\text { N368-K369 }\end{array}$ & $\begin{array}{l}\text { Zhang et al., } \\
2014\end{array}$ \\
\hline
\end{tabular}

TABLE 2 | (Continued)

\begin{tabular}{|c|c|c|c|c|}
\hline $\begin{array}{l}\text { Tau } \\
\text { fragment }\end{array}$ & $\begin{array}{l}\text { Description of } \\
\text { in vitro model }\end{array}$ & $\begin{array}{c}\text { Toxicity } \\
\text { mechanisms } \\
\text { of tau }\end{array}$ & $\begin{array}{l}\text { Associated } \\
\text { protease }\end{array}$ & References \\
\hline $256-441$ & $\begin{array}{c}\text { Primary } \\
\text { neurons } \\
\text { transfected } \\
\text { with adeno- } \\
\text { associated } \\
\text { viruses } \\
\text { encoding tau } \\
256-441\end{array}$ & Tau PHFs & $\begin{array}{l}\text { AEP cleaves at } \\
\text { N255-V256 }\end{array}$ & $\begin{array}{l}\text { Zhang et al., } \\
2014\end{array}$ \\
\hline $369-441$ & $\begin{array}{c}\text { Primary } \\
\text { neurons } \\
\text { transfected } \\
\text { with adeno- } \\
\text { associated } \\
\text { viruses } \\
\text { encoding tau } \\
\text { 369-441 }\end{array}$ & $\begin{array}{c}\text { Tau } \\
\text { aggregation } \\
\text { into PHFs with } \\
\text { a mixture of } \\
\text { fragments }\end{array}$ & $\begin{array}{l}\text { AEP cleaves at } \\
\text { N368-K369 }\end{array}$ & $\begin{array}{l}\text { Zhang et al., } \\
2014\end{array}$ \\
\hline $\begin{array}{l}243-441 \\
\text { (Tau- } \\
\text { CTF24, } 24 \\
\text { kDa) }\end{array}$ & $\begin{array}{l}\text { N2a cells } \\
\text { expressing } \\
\text { Tau-CTF24 and } \\
\text { Tau- FL and } \\
\text { treated with } \\
\text { Thioflavin S to } \\
\text { measure } \\
\text { aggregation } \\
\text { SH-SY5Y cells } \\
\text { expressing } \\
\text { Tau-CTF24 and } \\
\text { exposed to } \\
\text { various seeds } \\
\text { including } \\
\text { heparin- } \\
\text { induced } \\
\text { assembled Tau, } \\
\text { Tau- CTF24, or } \\
\text { sarkosyl- } \\
\text { insoluble pellets } \\
\text { from human } \\
\text { tauopathies }\end{array}$ & $\begin{array}{l}\text { Higher } \\
\text { aggregation, } \\
\text { reduced MT } \\
\text { stabilization } \\
\text { and MT } \\
\text { bundling } \\
\text { function in N2a } \\
\text { cells In } \\
\text { SH-SY5Y cells, } \\
\text { Tau-CTF24 } \\
\text { showed lower } \\
\text { seeding than } \\
\text { Tau-FL, but } \\
\text { Tau-CTF24 } \\
\text { showed greater } \\
\text { aggregation to } \\
\text { various seeds } \\
\text { in the ppt } \\
\text { fraction } \\
\text { compared to } \\
\text { Tau- FL }\end{array}$ & $\begin{array}{c}\text { Calpain } 1 \\
\text { cleaves at } \\
\text { R242-L243 }\end{array}$ & $\begin{array}{l}\text { Matsumoto } \\
\text { et al., } 2015\end{array}$ \\
\hline $1-391$ & $\begin{array}{c}\text { HEK-293FT cell } \\
\text { expression of } \\
\text { tau fragment }\end{array}$ & $\begin{array}{c}\text { Significant } \\
\text { insoluble tau in } \\
\text { cells that } \\
\text { expressed } \\
\text { 1-391, } \\
\text { enhanced } \\
\text { aggregation } \\
\text { due to deletion } \\
\text { of last } 50 \text { aa }\end{array}$ & $\begin{array}{l}\text { Unknown at } \\
\text { E391-1392 }\end{array}$ & Gu et al., 20 \\
\hline $1-421$ & $\begin{array}{c}\text { HEK-293FT cell } \\
\text { expression of } \\
\text { tau fragment }\end{array}$ & $\begin{array}{c}\text { Tau 1-421 } \\
\text { induced } \\
\text { mitochondria } \\
\text { fragmentation } \\
\text { and elevated } \\
\text { oxidative stress } \\
\text { levels } \\
\text { (Quintanilla } \\
\text { et al., 2009) }\end{array}$ & $\begin{array}{c}\text { Caspase-1, -3, } \\
-6,-7-, \text { and }-8 \\
\text { cleave at } \\
\text { D421-S422 }\end{array}$ & $\begin{array}{c}\text { Gamblin et al., } \\
\text { 2003; } \\
\text { Quintanilla } \\
\text { et al., 2009; Gu } \\
\text { et al., 2020 }\end{array}$ \\
\hline $51-391$ & $\begin{array}{l}\text { HEK-293FT cell } \\
\text { expression of } \\
\text { tau fragment }\end{array}$ & $\begin{array}{l}\text { Enhanced } \\
\text { aggregation } \\
\text { due to deletion } \\
\text { of last } 50 \text { aa }\end{array}$ & $\begin{array}{l}\text { N/A at T50- } \\
\text { P51,unknown } \\
\text { at E391-I392 }\end{array}$ & Gu et al., 2020 \\
\hline
\end{tabular}


TABLE 2 | (Continued)

\begin{tabular}{|c|c|c|c|c|}
\hline $\begin{array}{l}\text { Tau } \\
\text { fragment }\end{array}$ & $\begin{array}{l}\text { Description of } \\
\text { in vitro model }\end{array}$ & $\begin{array}{c}\text { Toxicity } \\
\text { mechanisms } \\
\text { of tau }\end{array}$ & $\begin{array}{l}\text { Associated } \\
\text { protease }\end{array}$ & References \\
\hline $51-421$ & $\begin{array}{l}\text { HEK-293FT cell } \\
\text { expression of } \\
\text { tau fragment }\end{array}$ & $\begin{array}{l}\text { No significant } \\
\text { increase in } \\
\text { ratio of RIPA } \\
\text { insoluble/ } \\
\text { soluble tau in } \\
\text { cells that } \\
\text { expressed tau } \\
51-421\end{array}$ & $\begin{array}{c}\text { N/A at } \\
\text { T50-P51, } \\
\text { Caspase -1, -3, } \\
-6,-7-\text {, and }-8 \\
\text { cleave at } \\
\text { D421-S422 }\end{array}$ & $\begin{array}{c}\text { Gamblin et al., } \\
\text { 2003; Gu et al., } \\
2020\end{array}$ \\
\hline $51-441$ & $\begin{array}{l}\text { HEK-293FT cell } \\
\text { expression of } \\
\text { tau fragment }\end{array}$ & $\begin{array}{l}\text { No significant } \\
\text { increase in } \\
\text { ratio of RIPA } \\
\text { insoluble/ } \\
\text { soluble tau in } \\
\text { cells that } \\
\text { expressed tau } \\
51-441\end{array}$ & $\mathrm{~N} / \mathrm{A}$ at $\mathrm{T} 50-\mathrm{P} 51$ & Gu et al., 2020 \\
\hline $151-391$ & $\begin{array}{c}\text { HEK-293FT cell } \\
\text { expression of } \\
\text { tau fragment }\end{array}$ & $\begin{array}{l}\text { High self- } \\
\text { aggregation } \\
\text { capacity }\end{array}$ & $\begin{array}{c}\text { ADAM-1 O } \\
\text { cleaves nearby } \\
\text { at I151-A152, } \\
\text { unknown at } \\
\text { E391-I392 }\end{array}$ & Gu et al., 2020 \\
\hline $151-421$ & $\begin{array}{l}\text { HEK-293FT cell } \\
\text { expression of } \\
\text { tau fragment }\end{array}$ & $\begin{array}{l}\text { Increases ratio } \\
\text { of RIPA-buffer } \\
\text { insoluble/ } \\
\text { soluble tau in } \\
\text { cells that } \\
\text { expressed tau } \\
151-421 \text {, } \\
\text { deletion of last } \\
20 \text { aa } \\
\text { decreased } \\
\text { ratio slightly }\end{array}$ & $\begin{array}{c}\text { ADAM-10 } \\
\text { cleaves at } \\
\text { 1151-A152, } \\
\text { Caspase -1, -3, } \\
-6,-7-\text {-, and -8 } \\
\text { cleave at } \\
\text { D421-S422 }\end{array}$ & $\begin{array}{c}\text { Gamblin et al., } \\
\text { 2003; Gu et al. } \\
2020\end{array}$ \\
\hline $151-441$ & $\begin{array}{l}\text { HEK-293FT cell } \\
\text { expression of } \\
\text { tau fragment }\end{array}$ & $\begin{array}{l}\text { Increase in } \\
\text { ratio of } \\
\text { RIPA-buffer } \\
\text { insoluble/ } \\
\text { soluble tau in } \\
\text { cells that } \\
\text { expressed tau } \\
151-421\end{array}$ & $\begin{array}{c}\text { ADAM-10 } \\
\text { cleaves nearby } \\
\text { at I151-A152 }\end{array}$ & $\begin{array}{l}\text { Henriksen } \\
\text { et al., 2013; Gu } \\
\text { et al., } 2020\end{array}$ \\
\hline $\begin{array}{l}\text { 153-441 } \\
\text { (Tau-A) }\end{array}$ & $\begin{array}{c}\text { Serum based } \\
\text { assay of tissue } \\
\text { from confirmed } \\
\text { AD post } \\
\text { mortem brains, } \\
\text { also Tg4510 } \\
\text { mice } \\
\text { expressing a } \\
\text { P301S } \\
\text { mutation }\end{array}$ & $\begin{array}{c}\text { Inverse } \\
\text { correlation } \\
\text { with MDRS, } \\
\text { indicating it is } \\
\text { related to loss } \\
\text { of cognitive } \\
\text { ability, elevated } \\
10 x \text { in Tg4510 } \\
\text { mice } \\
\text { compared to } \\
\text { control mice }\end{array}$ & $\begin{array}{l}\text { ADAM-10 } \\
\text { cleaves at } \\
\text { A152-T153 }\end{array}$ & $\begin{array}{l}\text { Henriksen } \\
\text { et al., } 2013\end{array}$ \\
\hline 231-391 & $\begin{array}{l}\text { HEK-293FT cell } \\
\text { expression of } \\
\text { tau fragment }\end{array}$ & $\begin{array}{c}\text { Low } \\
\text { expression of } \\
\text { tau 231-391, } \\
\text { which may } \\
\text { lead to } \\
\text { undetectable } \\
\text { aggregation of } \\
\text { tau 231-391, } \\
\text { deletion of last } \\
50 \text { aa did not } \\
\text { enhance ratio }\end{array}$ & $\begin{array}{c}\text { Calpain 1-2 } \\
\text { cleaves at } \\
\text { R230-T231, } \\
\text { unknown at } \\
\text { E391-I392 }\end{array}$ & $\begin{array}{c}\text { Garg et al., } \\
\text { 2010; Gu et al. } \\
2020\end{array}$ \\
\hline
\end{tabular}

(Continued)
TABLE 2 | (Continued)

\begin{tabular}{|c|c|c|c|c|}
\hline $\begin{array}{l}\text { Tau } \\
\text { fragment }\end{array}$ & $\begin{array}{l}\text { Description of } \\
\text { in vitro model }\end{array}$ & $\begin{array}{c}\text { Toxicity } \\
\text { mechanisms } \\
\text { of tau }\end{array}$ & $\begin{array}{l}\text { Associated } \\
\text { protease }\end{array}$ & References \\
\hline $231-421$ & $\begin{array}{c}\text { HEK-293FT cell } \\
\text { expression of } \\
\text { tau fragment }\end{array}$ & $\begin{array}{l}\text { Increase in ratio } \\
\text { of RIPA-buffer } \\
\text { insoluble/ } \\
\text { soluble tau in } \\
\text { cells that } \\
\text { expressed tau } \\
231-421\end{array}$ & $\begin{array}{c}\text { Calpain 1-2 } \\
\text { cleaves at } \\
\text { R230-T231, } \\
\text { Caspase-1, -3, } \\
-6,-7-\text {, and -8 } \\
\text { cleave at } \\
\text { D421-S422 }\end{array}$ & $\begin{array}{l}\text { Gamblin et al., } \\
\text { 2003; Garg } \\
\text { et al., 2010; Gu } \\
\text { et al., } 2020\end{array}$ \\
\hline $231-441$ & $\begin{array}{c}\text { HEK-293FT cell } \\
\text { expression of } \\
\text { tau fragment }\end{array}$ & $\begin{array}{l}\text { Increase in ratio } \\
\text { of RIPA-buffer } \\
\text { insoluble/ } \\
\text { soluble tau in } \\
\text { cells that } \\
\text { expressed tau } \\
231-441\end{array}$ & $\begin{array}{c}\text { Calpain 1-2 } \\
\text { cleaves at } \\
\text { R230-T231 } \\
\text { (Garg et al., } \\
\text { 2010) }\end{array}$ & $\begin{array}{c}\text { Garg et al., } \\
\text { 2010; Gu et al., } \\
2020\end{array}$ \\
\hline $\begin{array}{l}\text { 1-402 (Tau } \\
\Delta \text { Casp6) }\end{array}$ & $\begin{array}{c}\text { Post morterm } \\
\text { brain samples } \\
\text { from affected } \\
\text { AD temporal } \\
\text { and frontal } \\
\text { cortex }\end{array}$ & $\begin{array}{l}\text { Neotope } \\
\text { antibody to } \\
\text { caspase-6 } \\
\text { cleaved tau } \\
\text { detected } \\
\text { intracellular } \\
\text { tangles, } \\
\text { extracellular } \\
\text { tangles, } \\
\text { neuropil } \\
\text { threads, and } \\
\text { neurotic } \\
\text { plaques found } \\
\text { in temporal and } \\
\text { frontal cortex }\end{array}$ & $\begin{array}{c}\text { Caspase } 6 \text { at } \\
\text { D402-T403 }\end{array}$ & $\begin{array}{l}\text { Guo et al., } \\
2004\end{array}$ \\
\hline
\end{tabular}

This table includes tau fragments studied in vitro neuronal models that displayed neurotoxic molecular mechanisms. Some overlap among fragments studied in vivo transgenic mice models and in vitro neuronal models exist, such as tau 1-255 and tau 1-368 studied in Zhang et al. (2014), Tau 151-421 studied in Ozcelik et al. (2016), Gu et al. (2020), Tau 243-441 (Tau-CTF24) studied in Matsumoto et al. (2015), and Tau 26-230 studied in Corsetti et al. (2008).

compared to wild-type and $\mathrm{Lgmn}^{-/-}$mice. P301S/Lgmn ${ }^{-/-}$ mice showed ameliorated synapse lose compared to tau P301Stransgenic mice, indicating that Lgmn knockout prevents impairment in synaptic function caused by the tau mutation. Tau is cleaved by AEP in an age-dependent manner. At the age of 8 months, tau is cleaved by AEP when AEP expression is high. Memory function of P301S and P301S/Lgmn ${ }^{-/-}$mice was also tested using the Morris water maze. Tau P301S/Lgmn ${ }^{-/-}$ had greatly reduced learning deficits and improved memory retention compared to tau P301S-transgenic mice. AEP mediates neurotoxicity by generating at N255 and N368, cleaving two toxic fragments tau 1-255 and tau 1-368, confirmed by injection with AAVs encoding tau P301S and uncleavable AAVtauP301S/N255A/N368A. TauP301S/N255A/N368-expressing mice showed improved memory indicated by the decreased latency to find the platform in the water maze test and increased percentage of time in the target quadrant. Thus, synaptic function is preserved in mice expressing non-cleavable tau P301S and cleavage of tau is required for the effects of AEP (Zhang et al., 2014). 
Another group looked at the interaction between truncation and full-length human tau by generating an inducible mouse line (TAU62) expressing tau 151-421 (239 aa, human 3R) with a P301S tau mice (383 aa, human 4R) to produce the P301SxTau62 transgene mouse model (Ozcelik et al., 2016). Tau62 transgenic mice are generated by co-injection of two Thy 1.2 minigene-based constructs, which are obtained by inserting a tetracycline controlled transcriptional silencer (tTS) element complementary DNA. In the presence of doxycline tau151$421(\Delta \mathrm{tau})$ is expressed. In the absence of doxycycline, tTS binds to tetracycline-responsive element on the Thy1.2 minigene, preventing expression of tau151-421 ( $\Delta$ tau). Interestingly, P301SxTAU62 ${ }^{\text {on }}$ mice showed a drastic motor phenotype starting with gait ataxia at 9 days all the way to severe palsy by 3 weeks of age, which was recovered after 151-421 expression was halted at 3 weeks of age. Homozygous P301S tau mice developed immobilizing limb paralysis at approximately 5-7 months of age. P301SxTAU62 mice also displayed pretangle pathology and axonal transport deficiency, but many of these were also reversible with the cessation of tau 151-421 expression. Another transgenic mice that co-expressed $\Delta$ tau and full-length $4 \mathrm{R}$ human wt tau (ALZ17xTAU62 mice; Ozcelik et al., 2016) displayed nerve cell dysfunction, as well as motor palsy similar to the P301SxTAU62 mice. These findings demonstrate modified behavioral and cellular outcomes of $\Delta$ tau with and without other tau forms. Heterogeneity in tau fragment forms influences resultant deficits.

Tau 45-230 has also been studied in transgenic mice models (Lang et al., 2014). Generation of Tau 45-230-GTP transgenic mice was conducted by injecting the pronucleus of a singlecell fertilized C57BL/6J mouse embryo with the tau 45-230 transgene under control of the Thy 1.2 promoter. Similar to other transgenic studies, genotyping of tail biopsies by PCR and southern blot were done in order to identify the transgene positive founder mice. Neuronal loss was significant higher in 9and 12-month old Tg tau 45-230 mice compared to wild type controls. Levels of synaptophysin, a synaptic vesicle membrane protein, were significantly decreased as early as 6 months after birth in the transgenic mice compared to the wild type. Similar significant decreases were found in the NR2A and NR2B subunits of the NMDA receptors in the Tg tau 45-230 mice at the ages of 6 and 9 months. Tg tau 45-230 mice showed enhanced freezing behavior in fear tests. In the Morris water maze test, the tau $_{45-230}$ mice displayed mild memory defects compared to their age-matched wild type controls.

Another fragment called tau-CTF24 (243-441, $24 \mathrm{kDa})$ was increased with aging in a tauopathy model mice (Tg601) and was found to be produced by increased calpain activity in old Tg601 animals (Matsumoto et al., 2015). Tg601 mice express high levels of 2N4R under control of calcium/calmodulin-dependent protein kinase II promoter. Tau-CTF24 was found more abundantly in the brains of old Tg601 mice than in young Tg601 mice. When comparing non-tg mice and Tg601 mice at the same age, larger amounts of Tau-CTF24 were detected in the Tg601 group than in the non-Tg mice group. Similar results were replicated in vitro as well. Recombinant tau-CTF24 accelerated heparin-induced aggregation and lost the ability to promote
MT. Insoluble tau from diseased AD brains were introduced as seeds into SH-SY5Y cells and a large amount of insoluble tau was formed in cells overexpressing tau-CTF24 than in those expressing full tau. These results suggest that the absence of the N-terminal domain increases aggregation propensity and lowers MT assembly. Furthermore, lysates containing these tau-CTF24 inclusions propagated to naïve tau-expressing cells more efficiently than those containing full length tau inclusion. Importantly, western blots using the TauN, Tau5, Exon10v2, and TauC antibodies found the tau-CTF24 fragment in brain tissues from AD, familial FTLD with N279K mutation, PSP, and $\mathrm{CBD}$, respectively.

It must be realized that numerous tau fragments identified in animal models have unknown toxicity (examples are shown in Table 3). Among tau fragments found in brains from patients with tauopathy conditions, the contribution of each of the different tau fragments with respect to behavioral dysfunctions and neurotoxicity have not yet been fully defined. It will be important to define the full spectrum of tau fragments in tauopathies and define each of their biological activities.

Significantly, evidence for behavioral deficits and cellular toxicity resulting from expression of tau fragments in transgenic mouse models demonstrate that tau fragments participate in cognitive deficits of tauopathies.

\section{TAU TRUNCATION: N-TERMINAL AND C-TERMINAL CLEAVAGES MEDIATE AGGREGATION}

Tau is abnormally truncated at multiple sites, composing NFT and fibrillar aggregate structures in AD and related tauopathies (Ballatore et al., 2007). A wide spectrum of tau proteolytic fragments has been identified and characterized for neurotoxic and biological functions that may participate in tauopathies (Tables 1, 2; Quinn et al., 2018).

Enzyme-mediated truncation of tau at both the N-terminus (binds neural plasma membrane components) and the C-terminus (binds axonal MT) are facilitators of AD pathology. The deletion of the first 150 or 230 aa enhances tau site-specific phosphorylation, self-aggregation, and seeding, but the deletion of the first 50 aa did not produce significant effects. Similar deletion of the last 50 aa also promote self-aggregation and seeding, but not the deletion of the last 20 aa (Gu et al., 2020).

Several novel fragments consisting of tau 1-391, tau 51441, tau 51-421, tau 51-391, tau 151-441, tau-151-421, tau 151-391, tau 231-441, tau 231-421, and tau 231-391 were expressed in HEK-293T cells and were analyzed for toxicity by lactate dehydrogenase activity in the cell culture medium leaked from the cells, representing toxicity ( $\mathrm{Gu}$ et al., 2020). These fragments correspond to well-established truncation sites including K44-E45 by calpain-1 (Yang and Ksiezak-Reding, 1995), A152-T153 by ADAM10 (Henriksen et al., 2013), R230T231 by calpain 1-2 (Garg et al., 2010), caspase 3/6 by D421S422 (Zhao et al., 2016), and an unknown protease at E391I392. Tau 1-421 was also studied, which has been previously shown to aggravate mitochondrial fragmentation and elevated 
TABLE 3 | Tau fragments with unknown pathogenic functions.

\begin{tabular}{|c|c|c|c|c|}
\hline $\begin{array}{l}\text { Tau } \\
\text { fragment }\end{array}$ & $\begin{array}{c}\text { Model } \\
\text { description }\end{array}$ & $\begin{array}{c}\text { Toxicity } \\
\text { mechanisms }\end{array}$ & $\begin{array}{l}\text { Associated } \\
\text { protease }\end{array}$ & References \\
\hline $1-152$ & $\begin{array}{c}\text { Brain tissue } \\
\text { samples from } \\
\text { control Sprague } \\
\text { Dawley rats and } \\
\text { from Tg4510 tau } \\
\text { transgenic mice } \\
\text { overexpressing } \\
\text { the tau mutant } \\
\text { P301L and in } \\
\text { confirmed AD } \\
\text { post mortem } \\
\text { brain }\end{array}$ & Unknown & $\begin{array}{l}\text { ADAM-10 } \\
\text { cleaves at A } \\
\text { 152-T153 } \\
\text { (Henriksen } \\
\text { et al., 2013) }\end{array}$ & $\begin{array}{l}\text { Henriksen } \\
\text { et al., } 2013\end{array}$ \\
\hline $156-441$ & $\begin{array}{l}\text { Brain tissue from } \\
\text { frontal or temporal } \\
\text { regions of normal } \\
\text { human brains and } \\
\text { from perfused } \\
\text { Adult Wistar rat } \\
\text { brains, incubated } \\
\text { with and without } \\
\text { protease inhibitors }\end{array}$ & $\begin{array}{l}\text { Thrombin found } \\
\text { to cleave tau, } \\
\text { however, toxic } \\
\text { mechanisms of } \\
\text { fragment not } \\
\text { studied }\end{array}$ & $\begin{array}{c}\text { Thrombin } \\
\text { cleaves at } \\
\text { R155-G156 } \\
\text { (Arai et al., } \\
\text { 2005) }\end{array}$ & $\begin{array}{l}\text { Arai et al., } \\
2005\end{array}$ \\
\hline 156-209 & $\begin{array}{l}\text { Brain tissue from } \\
\text { frontal or temporal } \\
\text { regions of normal } \\
\text { human brains and } \\
\text { from perfused } \\
\text { Adult Wistar rat } \\
\text { brains, incubated } \\
\text { with and without } \\
\text { protease inhibitors }\end{array}$ & $\begin{array}{l}\text { Thrombin found } \\
\text { to cleave tau, } \\
\text { however, toxic } \\
\text { mechanisms of } \\
\text { fragment not } \\
\text { studied }\end{array}$ & $\begin{array}{c}\text { Thrombin } \\
\text { cleaves at } \\
\text { R155-G156 } \\
\text { and } \\
\text { R209-S210 } \\
\text { (Arai et al., } \\
\text { 2005) }\end{array}$ & $\begin{array}{l}\text { Arai et al., } \\
2005\end{array}$ \\
\hline $210-441$ & $\begin{array}{l}\text { Brain tissue from } \\
\text { frontal or temporal } \\
\text { regions of normal } \\
\text { human brains and } \\
\text { from perfused } \\
\text { Adult Wistar rat } \\
\text { brains, incubated } \\
\text { with and without } \\
\text { protease inhibitors }\end{array}$ & $\begin{array}{l}\text { Thrombin found } \\
\text { to cleave tau, } \\
\text { however, toxic } \\
\text { mechanisms of } \\
\text { fragment not } \\
\text { studied }\end{array}$ & $\begin{array}{c}\text { Thrombin } \\
\text { cleaves at } \\
\text { R209-S210 } \\
\text { (Arai et al., } \\
\text { 2005) }\end{array}$ & $\begin{array}{l}\text { Arai et al., } \\
2005\end{array}$ \\
\hline 210-230 & $\begin{array}{l}\text { Brain tissue from } \\
\text { frontal or temporal } \\
\text { regions of normal } \\
\text { human brains and } \\
\text { from perfused } \\
\text { Adult Wistar rat } \\
\text { brains, incubated } \\
\text { with and without } \\
\text { protease inhibitors }\end{array}$ & $\begin{array}{l}\text { Thrombin found } \\
\text { to cleave tau, } \\
\text { however, toxic } \\
\text { mechanisms of } \\
\text { fragment not } \\
\text { studied }\end{array}$ & $\begin{array}{c}\text { Thrombin } \\
\text { cleaves at } \\
\text { R209-S210 } \\
\text { and } \\
\text { R230-T231 } \\
\text { (Arai et al., } \\
\text { 2005) }\end{array}$ & $\begin{array}{c}\text { Arai et al., } \\
2005\end{array}$ \\
\hline $124-441$ & $\begin{array}{l}\text { LC-MS/MS } \\
\text { spectrometry of } \\
\text { peptides from } \\
\text { human brain } \\
\text { autopsy samples } \\
\text { and N1E-115 } \\
\text { mouse } \\
\text { neuroblastoma } \\
\text { cells }\end{array}$ & $\begin{array}{c}\text { None, cells } \\
\text { expressing tau } \\
\text { 124-441 had } \\
\text { increased } \alpha \text { - } \\
\text { tubulin } \\
\text { acetylation, } \\
\text { stronger binding } \\
\text { to MTs and less } \\
\text { sensitive to } \\
\text { depolymerization } \\
\text { compared to tau } \\
\text { 1-441 }\end{array}$ & Unknown & $\begin{array}{l}\text { Derisbourg } \\
\text { et al., } 2015\end{array}$ \\
\hline
\end{tabular}

(Continued)
TABLE 3 | (Continued)

\begin{tabular}{|c|c|c|c|c|}
\hline $\begin{array}{l}\text { Tau } \\
\text { fragment }\end{array}$ & $\begin{array}{c}\text { Model } \\
\text { description }\end{array}$ & $\begin{array}{c}\text { Toxicity } \\
\text { mechanisms }\end{array}$ & $\begin{array}{c}\text { Associated } \\
\text { protease }\end{array}$ & References \\
\hline $127-441$ & $\begin{array}{c}\text { LC-MS/MS } \\
\text { spectrometry of } \\
\text { peptides from } \\
\text { human brain } \\
\text { autopsy samples } \\
\text { and N1E-115 } \\
\text { mouse } \\
\text { neuroblastoma } \\
\text { cells }\end{array}$ & Unknown & Unknown & $\begin{array}{l}\text { Derisbourg } \\
\text { et al., } 2015\end{array}$ \\
\hline
\end{tabular}

This table summarizes identified tau fragments with unknown pathogenic functions, and unknown associated proteases. Tau fragments with unknown toxicity mechanisms and unknown associated proteases were identified in human brain and mouse neuroblastoma cells by Derisbourg et al. (2015), using LC-MS/MS, and include tau fragments 127-441, 172-441, 174-441, 224-441, 238-441, 240-441, 259-441, 261-441, 280-441, 306-41, 309-441, 311-441, and 331-441.

oxidative stress levels (Quintanilla et al., 2009). Tau aggregation was tested by overexpression of aforementioned HA-tagged tau fragments in the HEK-293FT cells which were separated by RIPA buffer-soluble and -insoluble fractions and analyzed the ratios of proteins in RIPA buffer-insoluble/soluble tau using western blot developed with anti HA to quantify concentration. Deletion of the first 50 aa did not affect insoluble/soluble tau ratios, regardless of C-terminal truncations, however, deletion of the first 150 aa significantly increased the ratio in Tau441 and Tau421 forms, but not in Tau391 forms. Deletion of the C-terminal 20 aa did not affect ratios of insoluble/soluble tau, however, deletion of the last 50 aa significant increased aggregation regardless of $\mathrm{N}$-terminal truncations except for Tau231 forms. It was also found that oligomeric tau induces tau aggregation in HEK293FT cells that were treated and analyzed using the same RIPA buffer-insoluble/soluble ratios of truncated tau. Specifically, Tau 151-391 appeared to have the highest self-aggregation activity and binding to oligomeric tau.

$\mathrm{N}$-terminal tau, which interacts with the plasma membrane (Brandt et al., 1995), is secreted and mediates neurotoxicity at synapses (Sayas et al., 2019; Amadoro et al., 2020; Brunello et al., 2020) demonstrated in tauopathy models. $\mathrm{NH}_{2}$-truncated tau fragments of $20-22 \mathrm{kDa}$ have been identified as CSF biomarkers of neurodegenerative diseases and have also been found in mitochondria extracted from cryopreserved synaptosomes (Amadoro et al., 2014;, 2020). A $\beta$ oligomers activated the production of $20-22 \mathrm{kDa} \mathrm{NH}_{2}$-terminal tau fragments in human SHSY5Y neuroblastoma and rat hippocampal neurons (Uberti et al., 1997; Amadoro et al., 2010). In a similar experiment, subtoxic doses of tau $\mathrm{NH}_{2}$-terminal peptide (26-44 AA long), which is the minimal active moiety of the longer $20-22 \mathrm{kDa}$ tau peptides, was secreted into the parenchyma and provoked presynaptic deficits of glutamate release on hippocampal synaptosomes and altered $\mathrm{Ca}^{2+}$ dynamics (Florenzano et al., 2017). This tau 2644 fragment is derived from a $17 \mathrm{kDa}$ tau 26-230 fragment via cleavage at D25-Q26 by caspase-3 (Corsetti et al., 2008). Tau 26-44 is seen at the lower end of detection in CSF samples containing both normal and elevated total tau levels, however, 
the most quantifiable tau peptides in CSF contain the mid-region (aa 156-224), likely due to resistance of degradation by proteases (Barthélemy et al., 2016).

Other studies describe C-terminal truncated tau fragments as another mediator of $\mathrm{AD}$ pathology. Flow cytometry data showing synaptic terminals immunolabeled with the tau antibody HT7 highlight an increase in $20 \mathrm{kDa}$ tau fragments and tau dimers in AD synapses (Sokolow et al., 2015). Furthermore, only $15-25 \%$ of the synaptosomes were positive for intact C-terminal tau or fully functional MTBR, suggesting that a combination of release of different tau peptides from $\mathrm{AD}$ synapses and the phosphorylation of those C-terminal truncated fragments exacerbate tau aggregation and synaptic dysfunction Cleavage and propagation of tau may be due to high tau localization in synaptic terminals in the cortex (Sokolow et al., 2015).

Secretion of full-length and proteolytic tau fragments have been observed in a variety of models. Secretion of tau into the interstitial fluid (Yamada et al., 2011), and eventually into CSF or plasma can be used as a biomarker for AD disease progression (Shoji, 2019). Recently, novel endogenous tau fragments have been identified from CSF samples by immunoprecipitation and mass spectrometry (Cicognola et al., 2019). Several endogenous peptides ending at aa 123 or 224 were identified, which were prominent in the CSF.

\section{PROTEASES KNOWN TO GENERATE TOXIC TAU FRAGMENTS}

An attractive therapeutic approach for attenuating toxicity of tau associated with proteolysis includes inhibiting proteasesubstrate interactions, reducing protease expression and/or activity, and blocking actions of proteolytic tau fragments. There are a multitude of known tau fragments generated by defined tau cleavage sites, responsible proteases, toxic functions, and are used as biomarkers in disease (reviewed by Quinn et al., 2018). As examples, selected tau proteolytic fragments which have demonstrated roles in neurotoxicity and have at least one known responsible protease for its production are illustrated in Figure 4. Enzymes participating in tau proteolysis consist of caspases, calpains, cathepsins, as well as contributions by the serine proteases A1 (HtrA1), disintegrin, metalloproteinase $10(\mathrm{ADAM}-10)$, and asparagine endopeptidase (AEP; Quinn et al., 2018).

\section{Caspase Proteases}

Caspase proteases comprise a majority of enzymes involved in proteolysis of tau. Caspases have cysteine in their active site and cleave proteins only after aspartic acid (Asp, D) residues. Caspase members include caspase-2 (Zhao et al., 2016), caspase-3 (Gamblin et al., 2003), and caspase-6 (Horowitz et al., 2004). Over 13 different caspases have been discovered in humans and have been studied extensively (Van Opdenbosch and Lamkanfi, 2019). Many different neurotoxic fragments cleaved by caspases have been detected in mice and cell culture models as well as from patients in serum or CSF. In rTg5410 tau mice, caspase2 cleaved tau at D134-L315 to result in the production of a N-terminal tau1-314 ( $\Delta$ tau314) fragment. The $\Delta$ tau314 fragment promotes tau aggregation in dendritic spines (Zhao et al., 2016).

Effector caspase-3, activated by caspase-2, cleaves at D25Q26 (Corsetti et al., 2008) as well as D421-S422 (Gamblin et al., 2003). Caspase-3 cleavage at D421-S422 results in Tau 1-421 (Tau-C) which is linked to AD and PSP (Gamblin et al., 2003; Zhao et al., 2015). Activation of caspase-3 by a pro-apoptotic activator protein called appoptosin leads to cell death; thus, downregulation of appoptosin can prevent cell death (Zhang et al., 2012).

Caspase-6 cleaves tau at D402-T403 and produces a Tau 1-402 fragment which has been used as biomarker for $\mathrm{AD}$ in CSF (Ramcharitar et al., 2013; LeBlanc et al., 2014; Quinn et al., 2018). Caspase-6 activity was found to be elevated in the CA1 of the hippocampus and in the EC-II (LeBlanc et al., 2014) and is extremely abundant in both sporadic (Guo et al., 2004 and familial AD (Albrecht et al., 2009). Notably, increased caspase- 6 activity in the anterior olfactory nucleus (which is responsible for mediating signals from the olfactory bulb) is correlated with tau pathology in human $\mathrm{AD}$ olfactory bulb brain sections (Foveau et al., 2016). Interestingly, tau pathology is common in the olfactory bulb of $\mathrm{AD}$ and Lewis body disease but is minimal or absent in PSP and CBD (Tsuboi et al., 2003).

\section{Calpain Proteases}

Calpains are a second class of calcium-activated cytosolic cysteine proteases that mediate tau proteolysis. These include calpain-1 (Yang and Ksiezak-Reding, 1995; Park and Ferreira, 2005) and calpain-2 (Garg et al., 2010). Dysregulation of calcium homeostasis has been proposed to induce abnormal activation of calpains (Suzuki et al., 2004). Calpain-1 participates in several neurodegenerative diseases including Huntington's disease and Parkinson's disease (PD; Siklos et al., 2015) and represents a potential therapeutic target.

Research on calpain has examined the $17 \mathrm{kDA}$ tau fragment spanning residues 45-230 that is produced by calpain-1 via cleavage at K44-E45 (Yang and Ksiezak-Reding, 1995) and calpain-1 and -2 at R230-T231 (Garg et al., 2010). Mouse models overexpressing Tau 45-230 show significantly increased hippocampal death, synaptic loss as early as 6 months, and behavioral abnormalities (Lang et al., 2014). These deficits were also accompanied by alterations in NMDA receptor signaling. More recently, Tau 45-230 mice displayed impaired anterograde and retrograde organelle transport (Afreen et al., 2017). Another cytotoxic fragment, Tau 243-441, was found to be generated by calpain-1; this Tau 243-441 fragment increased in aging mice, propagated to other tau expressing cells (Matsumoto et al., 2015), and initiated degradation of nicotinic acetylcholine receptor subunit $\alpha 4$ (Yin et al., 2016). Calpain-2 has been found to cleave tau at multiple sites that are primarily located in the C-terminal half of the tau protein (Yang and Ksiezak-Reding, 1995). 


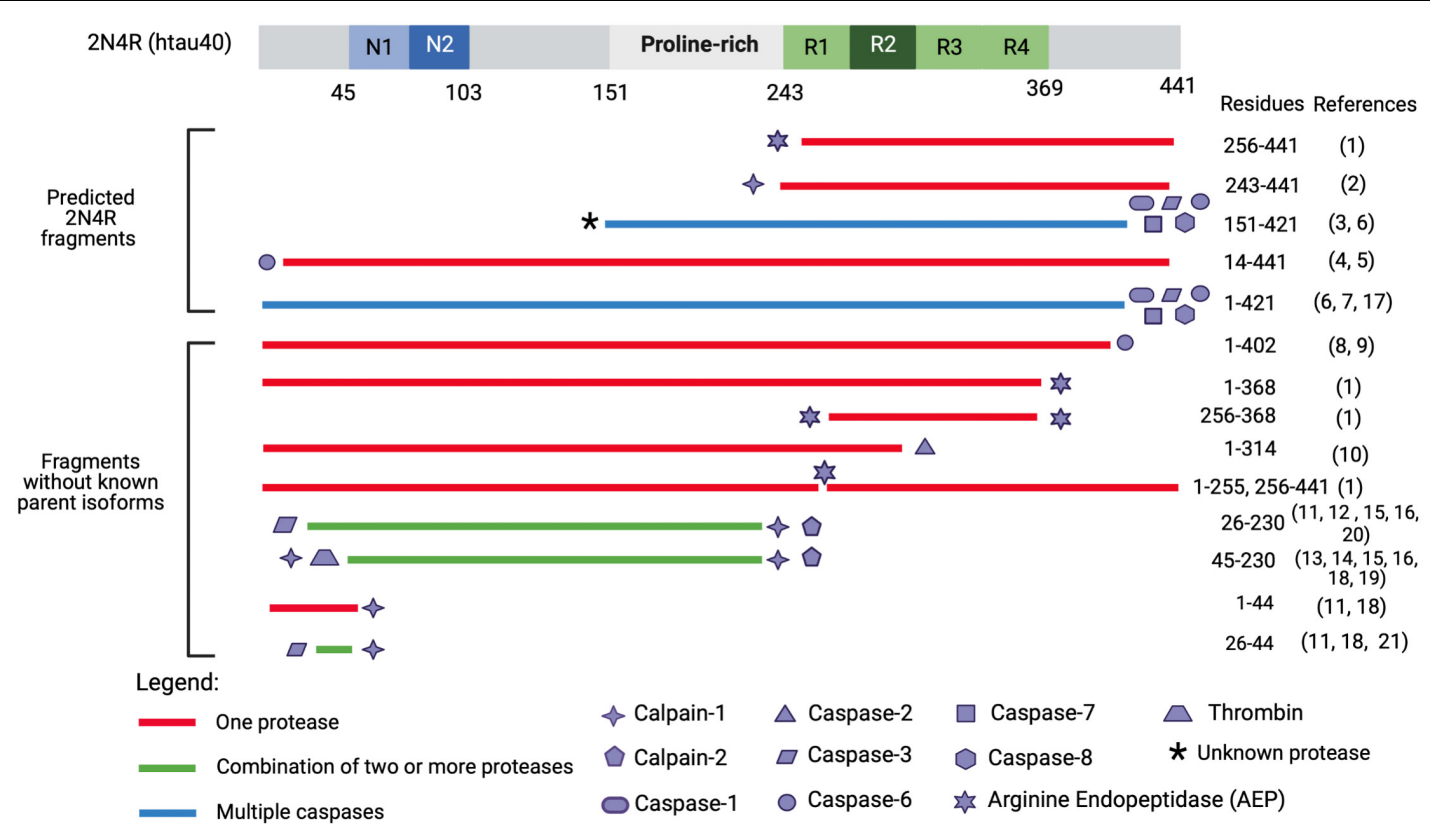

FIGURE 4 | Tau fragments found in in vitro and in vivo models with pathogenic functions and processing proteases. This figure illustrates a select group of tau proteolytic fragments which have been shown to have to drive neurotoxicity in either in vitro or in vivo models (correlated with Tables 1, 2) and have a known responsible protease. Precipitating proteases are represented by shape symbols and tau fragments are colored coded based on whether they are cleaved by one or multiple proteases. The top fragments are hypothesized to derive from $2 \mathrm{~N} 4 \mathrm{R}$ isoform as they extend past aa 410, which is the termination point of the second largest tau isoform, 2N3R. Several fragments from Gu et al. (2020) including tau 51-391, tau 51-421, tau 51-441, tau 151-391, tau 231-391, tau 231-421, and tau 231-441 are not included as they were engineered through recombinant plasmid techniques and have not been identified endogenously. Tau 187-441 and tau 1-391 are excluded as it is unclear which proteases cleaves at G186-E187. Tau 1-156 is excluded as it is unclear if thrombin, which cleaves at R155-G156 (Arai et al., 2005) also cleaves at G156-A157. The * symbol indicates that it is unknown what protease cleaves at the N-terminus of tau 151-421. Tau proteolytic fragments have not been studied to precipitate in up-regulated concentrations from 3R-or 4R-isoforms, and it is unclear whether 3R-or 4R-isoforms more toxic fragments. Reference numbers in parentheses refer to cited articles as follows: (1) Zhang et al. (2014); (2) Matsumoto et al. (2015); (3) Ozcelik et al. (2016); (4) Horowitz et al. (2004); (5) Horowitz et al. (2004); (6) Gamblin et al. (2003); (7) Quintanilla et al. (2009); (8) Foveau et al. (2016); (9) Guo et al. (2004); (10) Zhao et al. (2016); (11) Amadoro et al. (2006); (12) Amadoro et al. (2012); (13) Lang et al. (2014); (14) Afreen et al. (2017); (15) Park and Ferreira (2005); (16) Garg et al. (2010); (17) Matthews-Roberson et al. (2008); (18) Yang and Ksiezak-Reding (1995); (19) Arai et al. (2005); (20) Corsetti et al. (2008); and (21) Florenzano et al. (2017).

\section{Cathepsin Proteases}

Cathepsins represent a third class of proteases known to produce neurotoxic tau fragments. Cathepsins are lysosomal proteases utilized for normal protein degradation. However, in many neurodegenerative diseases including tauopathies, abnormal lysosomal leakage results in translocation of cathepsin proteases from the lysosome to the cytosol (Hook et al., 2020). Thus, lysosomal leakage may be a cellular event occurring prior to tau aggregation and NFT or fibrillary aggregate production (Yamashima, 2013; Hook et al., 2020). Several key cathepsins include cathepsins B, D, and L. Cathepsin B is associated with proteolytic tau cleavage and intracellular aggregation; cathepsin B is elevated and accumulates at amyloid plaques of $\mathrm{AD}$ brain (Cataldo and Nixon, 1990; Li et al., 1993). Another cathepsin, cathepsin D, participates in tau cleavages occurring at F8-E9, M419-V420, L436-A437, D34G161, P200-K257, and K267-D358 (Kenessey et al., 1997). Cathepsin L cleaved tau near the C-terminal region of tau, generating two highly amyloidogenic tau fragments. Cathepsin $\mathrm{L}$ cleaves at K245-S258, producing tau 258-372 (F1), then subsequently cleaves at V363-P364 to produce tau 258-363 (F2). Cathepsin L then cleaves tau 258-363 at I360-T361 to produce tau 258-360 (F3). F1 did not fully enter the lysosome but rather remained associated with lysosomal membranes (Wang et al., 2009).

\section{HtrA1, AEP, ADAM-10, and Thrombin}

Additional proteases have been found to cleave tau which include human high-temperature requirement serine protease A1 (HtrA1), metalloproteinase 10 (ADAM-10), AEP, and thrombin.

High-temperature requirement serine protease A1 is an ATPindependent serine protease (Quinn et al., 2018) and cleaves between A239 and V399, with preference for leucine, valine, and isoleucine. These cleavages degrade tau and result in 45 different fragments ranging from 1 to 22 aa residues in length. These tau degradative cleavages are associated with low levels of tau and its aggregates in the AD frontal cortex (Tennstaedt et al., 2012). Increased expression of HtRA1 may confer beneficial tau degradation and prevention of NFTs and fibrillar aggregates (Poepsel et al., 2015). HtrAl is also responsible for cleavage of the lipid transporting apolipoprotein E (ApoE) family, one of the major genetic risk factors for sporadic AD (Chu et al., 2016).

The lysosomal protease AEP, also known as legumain (LGMN), is synthesized as a zymogen (pro-LGMN, $36 \mathrm{kDa}$ ) 
and is auto-catalytically processed by sequential removal of $\mathrm{C}$ - and N-terminal pro-peptides to generate a $36 \mathrm{kDa}$ active LGMN enzyme at acidic pH (Dall and Brandstetter, 2016). AEP is active in acidic conditions within lysosomes, but lysosomal leakage results in translocation of AEP into the cytoplasm where it cleaves inhibitor 2 of protein phosphatase 2A (I2PP2A) that leads to increased tau hyperphosphorylation in AD (Basurto-Islas et al., 2018). Cleavage by other proteases is not necessary for AEP processing of tau and AEP cleavage is unaffected by hyperphosphorylation of tau (Zhang et al., 2014). AEP is phosphorylated and activated by serine-arginine protein kinase 2 (Wang et al., 2017) that leads to downstream interaction with tau (Zhang et al., 2014), alpha-synuclein (Zhang et al., 2017), and APP (Zhang et al., 2015). Tau cleavage by AEP occurs at N255-V256 and N368-K369 and results in five prominent tau fragments consisting of tau 1-255, tau 1-368, tau 256-368, tau 256-441, and tau 369-441. Among these fragments, Tau 1-368 and Tau 256-368 were particularly noteworthy for driving apoptosis in primary rat neurons and form PHF (Zhang et al., 2014). AEP is activated during the aging process (Zhang et al., 2014). A $\beta$ oligomers elicited AEP and tau fragmentation in dose-dependent manners in primary rat neuronal cultures, suggesting that $\mathrm{A} \beta$ provokes AEP activation (Zhang et al., 2014).

Another protease associated with tau is ADAM-10, a zinc metalloprotease that generates tau 153-441 (Tau A) by cleaving tau at A152-T153 (Henriksen et al., 2013). Upregulation of ADAM-10 cleaves APP into non-amyloidogenic species (reviewed by Andrew et al., 2016) but it is unclear still how membrane-bound ADAM-10 interacts with intracellular tau and whether tau 153-441 has any physiologic relevance to $\mathrm{AD}$ (Quinn et al., 2018).

Thrombin is a serine protease that is a component of the coagulation cascade (Chesser et al., 2013) and has been shown to be elevated in microvessels from $\mathrm{AD}$ brains compared to microvessels from control brains (Yin et al., 2010). Treatment of HT22 cells with thrombin resulted in formation of thioflavin$\mathrm{S}$ positive tau aggregates within $24 \mathrm{~h}$, followed by increase in cell death at $72 \mathrm{~h}$, but it is unclear how exogenous thrombin alters tau (Suo et al., 2003). However, thrombin has been found to cleave tau (Arai et al., 2005) at multiple arginine and lysine sites including: R155-G156, R209-S210, R230-T231, K257-S258, and K340-S341 and temporally, initial cleavage occurs at R155G156 (Arai et al., 2005). When tau was phosphorylated by glycogen synthase kinase-3beta, most of the proteolytic processes were inhibited, except for the first cleavage at R155-G156. Additionally, $\mathrm{PHF}$ prepared from $\mathrm{AD}$ brains were more resistant to thrombin proteolysis than following dephosphorylation by alkaline phosphatase (Arai et al., 2005). Thrombin-cleaved tau fragments have yet to be found in $\mathrm{AD}$ brains and other tauopathies, however.

Alternatively, thrombin may act as a neurotoxin by activating intracellular signaling cascades causing neurite retraction and stimulating apoptosis at high doses (Turgeon et al., 1998; De Luca et al., 2017). Thrombin is also a coagulation factor that plays a role neuroinflammatory diseases such as MS (Göbel et al., 2018). Furthermore, thrombin has been suggested to act as a mediator of vascular dysfunction and inflammation in both the periphery and the central nervous system, contributing to $\mathrm{AD}$ by inducing endothelial dysfunction of the blood brain barrier, microglia, astrocytes, and neurons (Iannucci et al., 2020).

\section{Unknown Proteases for Production of Tau Fragments}

It must be realized that while numerous tau fragments have been identified in tauopathy conditions in human brain, animal models, and cellular conditions, the proteases responsible for generating many of these tau fragments are unknown. It will be important to determine the proteases that generate toxic tau fragments, and also compare the relative toxicities of the spectrum of tau fragments.

\section{THE MISSING LINK: RELATIONSHIP AMONG TAU ISOFORMS, PROTEASES, AND PROTEOLYTIC FRAGMENTS THAT DRIVE NEUROTOXICITY}

Current studies have not provided the full details of which parent tau isoform(s), with or without mutations, serve as the source of specific tau fragments (Figure 3). Rather, identified diseaserelated tau fragments have been described with respect to the largest 2N4R (hT40) tau isoform (Figure 4). Among identified disease-exacerbating tau fragments, we propose that several fragments belong exclusively to the $2 \mathrm{~N} 4 \mathrm{R}$ isoform based on the presence of their $\mathrm{C}$-terminus containing residues beyond amino acid 410, which include a C-terminal truncated fragment 1-421, and several N-terminal truncated fragments: 14-441, 51-441, 51421, 124-441, 151-421, 151-441, 156-441, 231-441, 231-421, 243-441, 369-441, 403-441, and 422-441. Full determination of the mechanisms for proteolytic processing of each tau isoform has been challenging for several reasons consisting of: (1) the 6 tau isoforms share common sequence domains which requires detailed mass spectrometry analyses through multiple strategies to define the isoform origin of tau fragments, (2) the status of phosphorylated or non-phosphorylated tau isoforms and tauderived fragments as substrates for proteolysis has not been well defined, and (3) the sensitivity of peptide identification methods by immunochemical, mass spectrometry, and others differ greatly. Detailed understanding of the proteases that convert each of the tau isoforms into toxic fragments will be important to develop inhibitors of target proteases that may attenuate production of the most neurotoxic tau fragments that underlie specific tauopathy brain disorders.

\section{CONCLUSION}

Protease-mediated tau truncation is an important PTM which drives behavioral dysfunction and neurodegeneration in a tau fragment-dependent manner. While numerous tau fragments have been identified, knowledge of the proteolytic steps that convert each parent tau isoform into specific truncated tau fragments has not yet been fully defined. Therefore, the main 
conclusion of this review is that novel, intensive research is needed to define the details of the proteolytic steps utilized to convert each tau isoform into the most toxic fragments that drive behavioral deficits and tau pathology. Future advancement in understanding of the proteolytic processing mechanisms of tau isoforms can lead to new protease targeted drug strategies to prevent the formation of toxic tau fragments and ameliorate tauopathy disease deficits.

\section{AUTHOR CONTRIBUTIONS}

$\mathrm{BB}$ and $\mathrm{VH}$ planned the topics covered and wrote the manuscript. $\mathrm{VH}$ guided the overall outline, writing, and editing of the

\section{REFERENCES}

Afreen, S., Riherd Methner, D. N., and Ferreira, A. (2017). Tau45-230 association with the cytoskeleton and membrane-bound organelles: functional implications in neurodegeneration. Neuroscience 362, 104-117. doi: 10.1016/j.neuroscience. 2017.08.026

Albrecht, S., Bogdanovic, N., Ghetti, B., Winblad, B., and LeBlanc, A. C. (2009). Caspase-6 activation in familial alzheimer disease brains carrying amyloid precursor protein or presenilin i or presenilin II mutations. J. Neuropathol. Exp. Neurol. 68, 1282-1293. doi: 10.1097/NEN.0b013e3181c $1 \mathrm{da} 10$

Alonso, A. C., Zaidi, T., Grundke-Iqbal, I., and Iqbal, K. (1994). Role of abnormally phosphorylated tau in the breakdown of microtubules in Alzheimer disease. Proc. Natl. Acad. Sci. U. S. A. 91, 5562-5566. doi: 10.1073/pnas.91.12.5562

Alquezar, C., Arya, S., and Kao, A. W. (2020). Tau Post-translational Modifications: dynamic Transformers of Tau Function, Degradation, and Aggregation. Front. Neurol. 11:595532. doi: 10.3389/fneur.2020.595532

Amadoro, G., Ciotti, M. T., Costanzi, M., Cestari, V., Calissano, P., and Canu, N. (2006). NMDA receptor mediates tau-induced neurotoxicity by calpain and ERK/MAPK activation. Proc. Natl. Acad. Sci. U. S. A. 103, 2892-2897. doi: 10.1073/pnas.0511065103

Amadoro, G., Corsetti, V., Atlante, A., Florenzano, F., Capsoni, S., Bussani, R., et al. (2012). Interaction between NH(2)-tau fragment and A $\beta$ in Alzheimer's disease mitochondria contributes to the synaptic deterioration. Neurobiol. Aging. 33, e1-25. doi: 10.1016/j.neurobiolaging.2011.08.001

Amadoro, G., Corsetti, V., Sancesario, G. M., Lubrano, A., Melchiorri, G., Bernardini, S., et al. (2014). Cerebrospinal fluid levels of a 20-22 kDa $\mathrm{NH} 2$ fragment of human tau provide a novel neuronal injury biomarker in Alzheimer's disease and other dementias. J. Alzheimer. Dis. 42, 211-226. doi: 10.3233/JAD- 140267

Amadoro, G., Corsetti, V., Stringaro, A., Colone, M., D’Aguanno, S., Meli, G., et al. (2010). NH2 tau fragment targets neuronal mitochondria at AD synapses: possible implications for neurodegeneration. J. Alzheimer. Dis. 21, 445-470. doi: 10.3233/JAD-2010-100120

Amadoro, G., Latina, V., Corsetti, V., and Calissano, P. (2020). N-terminal tau truncation in the pathogenesis of Alzheimer's disease (AD): developing a novel diagnostic and therapeutic approach. Biochim. Biophys. Acta Mol. Basis Dis. 1866:165584. doi: 10.1016/j.bbadis.2019.165584

Anderson, D. B., Zanella, C. A., Henley, J. M., and Cimarosti, H. (2017). Sumoylation: implications for Neurodegenerative Diseases. Adv. Exp. Med. Biol. 963, 261-281. doi: 10.1007/978-3-319-50044-7_16

Andreadis, A. (2005). Tau gene alternative splicing: expression patterns, regulation and modulation of function in normal brain and neurodegenerative diseases. Biochim. Biophys. Acta 1739, 91-103. doi: 10.1016/j.bbadis.2004.08.010

Andrew, R. J., Kellett, K. A., Thinakaran, G., and Hooper, N. M. A. (2016). Greek Tragedy: the Growing Complexity of Alzheimer Amyloid Precursor Protein Proteolysis. J. Biol. Chem. 291, 19235-19244. doi: 10.1074/jbc.R116.746032

Arai, T., Guo, J. P., and McGeer, P. L. (2005). Proteolysis of non-phosphorylated and phosphorylated tau by thrombin. J. Biol. Chem. 280, 5145-5153. doi: 10.1074/jbc.M409234200 manuscript. Both authors contributed to the article and approved the submitted version.

\section{FUNDING}

This research was supported by NIH grant R01NS094597 (awarded to $\mathrm{VH}$ ).

\section{ACKNOWLEDGMENTS}

Figures were created with BioRender.com.

Arnold, C. S., Johnson, G. V., Cole, R. N., Dong, D. L., Lee, M., and Hart, G. W. (1996). The microtubule-associated protein tau is extensively modified with O-linked N-acetylglucosamine. J. Biol. Chem. 271, 28741-28744. doi: 10.1074/ jbc.271.46.28741

Arrasate, M., Pérez, M., Armas-Portela, R., and Avila, J. (1999). Polymerization of tau peptides into fibrillar structures. The effect of FTDP-17 mutations. FEBS Lett. 446, 199-202. doi: 10.1016/S0014-5793(99)00210-0

Ballatore, C., Lee, V. M., and Trojanowski, J. Q. (2007). Tau-mediated neurodegeneration in Alzheimers disease and related disorders. Nat. Rev. Neurosci. 8, 663-672. doi: 10.1038/nrn2194

Bancher, C., Grundke-Iqbal, I., Iqbal, K., Fried, V. A., Smith, H. T., and Wisniewski, H. M. (1991). Abnormal phosphorylation of tau precedes ubiquitination in neurofibrillary pathology of Alzheimer disease. Brain Res. 539, 11-18. doi: 10.1016/0006-8993(91)90681-K

Barbier, P., Zejneli, O., Martinho, M., Lasorsa, A., Belle, V., Smet-Nocca, C., et al. (2019). Role of Tau as a Microtubule-Associated Protein: structural and Functional Aspects. Front. Aging Neurosci. 11:204. doi: 10.3389/fnagi.2019. 00204

Barghorn, S., Zheng-Fischhöfer, Q., Ackmann, M., Biernat, J., von Bergen, M., Mandelkow, E. M., et al. (2000). Structure, microtubule interactions, and paired helical filament aggregation by tau mutants of frontotemporal dementias. Biochemistry 39, 11714-11721. doi: 10.1021/bi000850r

Barthélemy, N. R., Fenaille, F., Hirtz, C., Sergeant, N., Schraen-Maschke, S., Vialaret, J., et al. (2016). Tau Protein Quantification in Human Cerebrospinal Fluid by Targeted Mass Spectrometry at High Sequence Coverage Provides Insights into Its Primary Structure Heterogeneity. J. Proteome Res. 15, 667-676. doi: 10.1021/acs.jproteome.5b01001

Basurto-Islas, G., Gu, J. H., Tung, Y. C., Liu, F., and Iqbal, K. (2018). Mechanism of Tau Hyperphosphorylation Involving Lysosomal Enzyme Asparagine Endopeptidase in a Mouse Model of Brain Ischemia. J. Alzheimer. Dis. 63, 821-833. doi: 10.3233/JAD-170715

Biernat, J., Gustke, N., Drewes, G., Mandelkow, E. M., and Mandelkow, E. (1993). Phosphorylation of Ser262 strongly reduces binding of tau to microtubules: distinction between PHF-like immunoreactivity and microtubule binding. Neuron 11, 153-163. doi: 10.1016/0896-6273(93)90279-Z

Bondulich, M. K., Guo, T., Meehan, C., Manion, J., Rodriguez Martin, T., Mitchell, J. C., et al. (2016). Tauopathy induced by low level expression of a human brain-derived tau fragment in mice is rescued by phenylbutyrate. Brain 139, 2290-2306. doi: 10.1093/brain/aww137

Brandt, R., Léger, J., and Lee, G. (1995). Interaction of tau with the neural plasma membrane mediated by tau's amino-terminal projection domain. J. Biol. 131, 1327-1340. doi: 10.1083/jcb.131.5.1327

Brion, J. P., Octave, J. N., and Couck, A. M. (1994). Distribution of the phosphorylated microtubule-associated protein tau in developing cortical neurons. Neuroscience 63, 895-909. doi: 10.1016/0306-4522(94) 90533-9

Brunello, C. A., Merezhko, M., Uronen, R. L., and Huttunen, H. J. (2020). Mechanisms of secretion and spreading of pathological tau protein. Cell. Mol. Life Sci. 77, 1721-1744. doi: 10.1007/s00018-01903349-1 
Buée, L., and Delacourte, A. (1999). Comparative biochemistry of tau in progressive supranuclear palsy, corticobasal degeneration, FTDP-17 and Pick's disease. Brain Pathol. 9, 681-693. doi: 10.1111/j.1750-3639.1999.tb00550.x

Caillet-Boudin, M. L., Buée, L., Sergeant, N., and Lefebvre, B. (2015). Regulation of human MAPT gene expression. Mol. Neurodegener. 10:28. doi: 10.1186/ s13024-015-0025-8

Cataldo, A. M., and Nixon, R. A. (1990). Enzymatically active lysosomal proteases are associated with amyloid deposits in Alzheimer brain. Proc. Natl. Acad. Sci. U. S. A. 87, 3861-3865. doi: 10.1073/pnas.87.10.3861

Chen, X., Li, Y., Wang, C., Tang, Y., Mok, S. A., Tsai, R. M., et al. (2020). Promoting tau secretion and propagation by hyperactive p300/CBP via autophagylysosomal pathway in tauopathy. Mol. Neurodegener. 15:2. doi: 10.1186/ s13024-019-0354-0

Chesser, A. S., Pritchard, S. M., and Johnson, G. V. (2013). Tau clearance mechanisms and their possible role in the pathogenesis of Alzheimer disease. Front. Neurol. 4:122. doi: 10.3389/fneur.2013.00122

Chu, Q., Diedrich, J. K., Vaughan, J. M., Donaldson, C. J., Nunn, M. F., Lee, K. F., et al. (2016). HtrA1 Proteolysis of ApoE In Vitro Is Allele Selective. J. Am. Chem. Soc. 138, 9473-9478. doi: 10.1021/jacs.6b03463

Cicognola, C., Brinkmalm, G., Wahlgren, J., Portelius, E., Gobom, J., Cullen, N. C., et al. (2019). Novel tau fragments in cerebrospinal fluid: relation to tangle pathology and cognitive decline in Alzheimer's disease. Acta Neuropathol. 137, 279-296. doi: 10.1007/s00401-018-1948-2

Cleveland, D. W., Hwo, S. Y., and Kirschner, M. W. (1977). Purification of tau, a microtubule-associated protein that induces assembly of microtubules from purified tubulin. J. Mol. Biol. 116, 207-225. doi: 10.1016/0022-2836(77)90213-3

Cohen, T. J., Constance, B. H., Hwang, A. W., James, M., and Yuan, C. X. (2016). Intrinsic Tau Acetylation Is Coupled to Auto-Proteolytic Tau Fragmentation. PLoS One 11:e0158470. doi: 10.1371/journal.pone.0158470

Combs, B., Mueller, R. L., Morfini, G., Brady, S. T., and Kanaan, N. M. (2019). Tau and Axonal Transport Misregulation in Tauopathies. Adv. Exp. Med. Biol. 1184, 81-95. doi: 10.1007/978-981-32-9358-8_7

Corsetti, V., Amadoro, G., Gentile, A., Capsoni, S., Ciotti, M. T., Cencioni, M. T., et al. (2008). Identification of a caspase-derived $\mathrm{N}$-terminal tau fragment in cellular and animal Alzheimer's disease models. Mol. Cell. Neurosci. 38, 381392. doi: 10.1016/j.mcn.2008.03.011

Derisbourg, M., Leghay, C., Chiappetta, G., Fernandez-Gomez, F. J., Laurent, C., Demeyer, D., et al. (2015). Role of the Tau N-terminal region in microtubule stabilization revealed by new endogenous truncated forms. Sci. Rep. 5:9659. doi: $10.1038 /$ srep09659

D'Souza, I., and Schellenberg, G. D. (2005). Regulation of tau isoform expression and dementia. Biochim. Biophys. Acta 1739, 104-115. doi: 10.1016/j.bbadis. 2004.08.009

D’Souza, I., Poorkaj, P., Hong, M., Nochlin, D., Lee, V. M., Bird, T. D., et al. (1999). Missense and silent tau gene mutations cause frontotemporal dementia with parkinsonism-chromosome 17 type, by affecting multiple alternative RNA splicing regulatory elements. Proc. Natl. Acad. Sci. U. S. A. 96, 5598-5603. doi: $10.1073 /$ pnas. 96.10 .5598

Dall, E., and Brandstetter, H. (2016). Structure and function of legumain in health and disease. Biochimie 122, 126-150. doi: 10.1016/j.biochi.2015.09.022

de Calignon, A., Polydoro, M., Suárez-Calvet, M., William, C., Adamowicz, D. H., Kopeikina, K. J., et al. (2012). Propagation of tau pathology in a model of early Alzheimer's disease. Neuron 73, 685-697. doi: 10.1016/j.neuron.2011.11.033

De Luca, C., Virtuoso, A., Maggio, N., and Papa, M. (2017). Neuro-Coagulopathy: blood Coagulation Factors in Central Nervous System Diseases. Int. J. Mol. Sci. 18:2128. doi: 10.3390/ijms 18102128

Drazic, A., Myklebust, L. M., Ree, R., and Arnesen, T. (2016). The world of protein acetylation. Biochim. Biophys. Acta 1864, 1372-1401. doi: 10.1016/j.bbapap. 2016.06.007

Falcon, B., Zivanov, J., Zhang, W., Murzin, A. G., Garringer, H. J., Vidal, R., et al. (2019). Novel tau filament fold in chronic traumatic encephalopathy encloses hydrophobic molecules. Nature 568, 420-423. doi: 10.1038/s41586-019-1026-5

Fitzpatrick, A. W. P., Falcon, B., He, S., Murzin, A. G., Murshudov, G., Garringer, H. J., et al. (2017). Cryo-EM structures of tau filaments from Alzheimer's disease. Nature 547, 185-190. doi: 10.1038/nature23002

Florenzano, F., Veronica, C., Ciasca, G., Ciotti, M. T., Pittaluga, A., Olivero, G., et al. (2017). Extracellular truncated tau causes early presynaptic dysfunction associated with Alzheimer's disease and other tauopathies. Oncotarget 8, 6474564778. doi: 10.18632/oncotarget.17371

Foveau, B., Albrecht, S., Bennett, D. A., Correa, J. A., and LeBlanc, A. C. (2016). Increased Caspase- 6 activity in the human anterior olfactory nuclei of the olfactory bulb is associated with cognitive impairment. Acta Neuropathol. Commun. 4:127. doi: 10.1186/s40478-016-0400-x

Franzmeier, N., Neitzel, J., Rubinski, A., Smith, R., Strandberg, O., Ossenkoppele, R., et al. (2020). Alzheimer's Disease Neuroimaging Initiative (ADNI). Functional brain architecture is associated with the rate of tau accumulation in Alzheimer's disease. Nat. Commun. 11:347. doi: 10.1038/s41467-019-1 4159-1

Fuster-Matanzo, A., Hernández, F., and Ávila, J. (2018). Tau Spreading Mechanisms; Implications for Dysfunctional Tauopathies. Int. J. Mol. Sci. 19:645. doi: $10.3390 / \mathrm{ijms} 19030645$

Gamblin, T. C., Chen, F., Zambrano, A., Abraha, A., Lagalwar, S., Guillozet, A. L., et al. (2003). Caspase cleavage of tau: linking amyloid and neurofibrillary tangles in Alzheimer's disease. Proc. Natl. Acad. Sci. U. S. A. 100, 10032-10037. doi: 10.1073/pnas. 1630428100

García-Sierra, F., Jarero-Basulto, J. J., Kristofikova, Z., Majer, E., Binder, L. I., and Ripova, D. (2012). Ubiquitin is associated with early truncation of tau protein at aspartic acid(421) during the maturation of neurofibrillary tangles in Alzheimer's disease. Brain Pathol. 22, 240-250. doi: 10.1111/j.1750-3639.2011. 00525.x

Garg, S., Timm, T., Mandelkow, E. M., Mandelkow, E., and Wang, Y. (2010). Cleavage of Tau by calpain in Alzheimer's disease: the quest for the toxic $17 \mathrm{kD}$ fragment. Neurobiol. Aging 32, 1-14. doi: 10.1016/j.neurobiolaging.2010.09.008

Gasparini, L., Terni, B., and Spillantini, M. G. (2007). Frontotemporal dementia with tau pathology. Neurodegener. Dis. 4, 236-253. doi: 10.1159/000101848

Göbel, K., Eichler, S., Wiendl, H., Chavakis, T., Kleinschnitz, C., and Meuth, S. G. (2018). The Coagulation Factors Fibrinogen, Thrombin, and Factor XII in Inflammatory Disorders-A Systematic Review. Front. Immunol. 9:1731. doi: 10.3389/fimmu.2018.01731

Goedert, M. (2005). Tau gene mutations and their effects. Mov. Disord. 20, S45-S52. doi: $10.1002 / \mathrm{mds} .20539$

Goedert, M., and Jakes, R. (1990). Expression of separate isoforms of human tau protein: correlation with the tau pattern in brain and effects on tubulin polymerization. EMBO J. 9, 4225-4230. doi: 10.1002/j.1460-2075.1990. tb07870.x

Goedert, M., and Spillantini, M. G. (2000). Tau mutations in frontotemporal dementia FTDP-17 and their relevance for Alzheimer's disease. Biochim. Biophys. Acta 1502, 110-121. doi: 10.1016/S0925-4439(00)00037-5

Goedert, M., and Spillantini, M. G. (2001). Tau gene mutations and neurodegeneration. Biochem. Soc. Symp. 2001, 59-71. doi: 10.1042/bss0670059

Goedert, M., Eisenberg, D. S., and Crowther, R. A. (2017). Propagation of Tau Aggregates and Neurodegeneration. Annu. Rev. Neurosci. 40, 189-210. doi: 10.1146/annurev-neuro-072116-031153

Goedert, M., Ghetti, B., and Spillantini, M. G. (2000). Tau gene mutations in frontotemporal dementia and parkinsonism linked to chromosome 17 (FTDP-17). Their relevance for understanding the neurogenerative process. Ann. N. Y. Acad. Sci. 920, 74-83. doi: 10.1111/j.1749-6632.2000.tb06 907.x

Goedert, M., Jakes, R., and Crowther, R. A. (1999). Effects of frontotemporal dementia FTDP-17 mutations on heparin-induced assembly of tau filaments. FEBS Lett. 450, 306-311. doi: 10.1016/S0014-5793(99)00508-6

Goedert, M., Jakes, R., Crowther, R. A., Six, J., Lübke, U., Vandermeeren, M., et al. (1993). The abnormal phosphorylation of tau protein at Ser-202 in Alzheimer disease recapitulates phosphorylation during development. Proc. Natl. Acad. Sci. U. S. A. 90, 5066-5070. doi: 10.1073/pnas.90.11.5066

Goedert, M., Spillantini, M. G., Potier, M. C., Ulrich, J., and Crowther, R. A. (1989). Cloning and sequencing of the cDNA encoding an isoform of microtubuleassociated protein tau containing four tandem repeats: differential expression of tau protein mRNAs in human brain. EMBO J. 8, 393-399. doi: 10.1002/j. 1460-2075.1989.tb03390.x

Goode, B. L., Chau, M., Denis, P. E., and Feinstein, S. C. (2000). Structural and functional differences between 3-repeat and 4-repeat tau isoforms. Implications for normal tau function and the onset of neurodegenetative disease. J. Biol. Chem. 275, 38182-38189. doi: 10.1074/jbc.M007489200 
Gu, J., Xu, W., Jin, N., Li, L., Zhou, Y., Chu, D., et al. (2020). Truncation of Tau selectively facilitates its pathological activities. J. Biol. Chem. 295, 13812-13828. doi: 10.1074/jbc.RA120.012587

Guo, H., Albrecht, S., Bourdeau, M., Petzke, T., Bergeron, C., and LeBlanc, A. C. (2004). Active caspase-6 and caspase-6-cleaved tau in neuropil threads, neuritic plaques, and neurofibrillary tangles of Alzheimer's disease. Am. J. Pathol. 165, 523-531. doi: 10.1016/S0002-9440(10)63317-2

Hanger, D. P., Anderton, B. H., and Noble, W. (2009). Tau phosphorylation: the therapeutic challenge for neurodegenerative disease. Trends Mol. Med. 15, 112-119. doi: 10.1016/j.molmed.2009.01.003

Hefti, M. M., Kim, S., Bell, A. J., Betters, R. K., Fiock, K. L., Iida, M. A., et al. (2019). Tau Phosphorylation and Aggregation in the Developing Human Brain. J. Neuropathol. Exp. Neurol. 78, 930-938. doi: 10.1093/jnen/nlz073

Henley, J. M., Craig, T. J., and Wilkinson, K. A. (2014). Neuronal SUMOylation: mechanisms, physiology, and roles in neuronal dysfunction. Physiol. Rev. 94, 1249-1285. doi: 10.1152/physrev.00008.2014

Henriksen, K., Wang, Y., Sørensen, M. G., Barascuk, N., Suhy, J., Pedersen, J. T., et al. (2013). An enzyme-generated fragment of tau measured in serum shows an inverse correlation to cognitive function. PLoS One 8:e64990. doi: 10.1371/ journal.pone.0064990

Hong, M., Zhukareva, V., Vogelsberg-Ragaglia, V., Wszolek, Z., Reed, L., Miller, B. I., et al. (1998). Mutation-specific functional impairments in distinct tau isoforms of hereditary FTDP-17. Science 282, 1914-1917. doi: 10.1126/science. 282.5395.1914

Hook, V., Yoon, M., Mosier, C., Ito, G., Podvin, S., Head, B. P., et al. (2020). Cathepsin B in neurodegeneration of Alzheimer's disease, traumatic brain injury, and related brain disorders. Biochim. Biophys. Acta Proteins Proteom. 1868:140428. doi: 10.1016/j.bbapap.2020.140428

Horowitz, P. M., Patterson, K. R., Guillozet-Bongaarts, A. L., Reynolds, M. R., Carroll, C. A., Weintraub, S. T., et al. (2004). Early N-terminal changes and caspase-6 cleavage of tau in Alzheimer's disease. J. Neurosci. 24, 7895-7902. doi: 10.1523/JNEUROSCI.1988-04.2004

Hu, W., Wu, F., Zhang, Y., Gong, C. X., Iqbal, K., and Liu, F. (2017). Expression of Tau Pathology-Related Proteins in Different Brain Regions: a Molecular Basis of Tau Pathogenesis. Front. Aging Neurosci. 9:311. doi: 10.3389/fnagi.2017.00311

Hutton, M., Lendon, C. L., Rizzu, P., Baker, M., Froelich, S., Houlden, H., et al. (1998). Association of missense and 5'-splice-site mutations in tau with the inherited dementia FTDP-17. Nature 393, 702-705. doi: 10.1038/31508

Iannucci, J., Renehan, W., and Grammas, P. (2020). Thrombin, a Mediator of Coagulation, Inflammation, and Neurotoxicity at the Neurovascular Interface: implications for Alzheimer's Disease. Front. Neurosci. 14:762. doi: 10.3389/ fnins.2020.00762

Iqbal, K., Liu, F., Gong, C. X., and Grundke-Iqbal, I. (2010). Tau in Alzheimer disease and related tauopathies. Curr. Alzheimer. Res. 7, 656-664. doi: 10.2174/ 156720510793611592

Iqbal, K., Zaidi, T., Bancher, C., and Grundke-Iqbal, I. (1994). Alzheimer paired helical filaments. Restoration of the biological activity by dephosphorylation. FEBS Lett. 349, 104-108. doi: 10.1016/0014-5793(94)00650-4

Irwin, D. J., Cohen, T. J., Grossman, M., Arnold, S. E., Xie, S. X., Lee, V. M., et al. (2012). Acetylated tau, a novel pathological signature in Alzheimer's disease and other tauopathies. Brain 135, 807-818. doi: 10.1093/brain/aws013

Izzu, P., Joosse, M., Ravid, R., Hoogeveen, A., Kamphorst, W., van Swieten, J. C., et al. (2000). Mutation-dependent aggregation of tau protein and its selective depletion from the soluble fraction in brain of P301LFTDP-17 patients. Hum. Mol. Genet. 9, 3075-3082. doi: 10.1093/hmg/9.20.3075

Jeganathan, S., von Bergen, M., Mandelkow, E. M., and Mandelkow, E. (2008). The natively unfolded character of tau and its aggregation to Alzheimer-like paired helical filaments. Biochemistry 47, 10526-10539. doi: 10.1021/bi800783d

Johnson, G. V. (2006). Tau phosphorylation and proteolysis: insights and perspectives. J. Alzheimer. Dis. 9, 243-250. doi: 10.3233/JAD-2006-9S326

Kenessey, A., Nacharaju, P., Ko, L. W., and Yen, S. H. (1997). Degradation of tau by lysosomal enzyme cathepsin D: implication for Alzheimer neurofibrillary degeneration. J. Neurochem. 69, 2026-2038. doi: 10.1046/j.1471-4159.1997. 69052026.x

Kolarova, M., García-Sierra, F., Bartos, A., Ricny, J., and Ripova, D. (2012). Structure and pathology of tau protein in Alzheimer disease. Int. J. Alzheimers. Dis. 2012:731526. doi: 10.1155/2012/731526
Lang, A. E., Riherd Methner, D. N., and Ferreira, A. (2014). Neuronal degeneration, synaptic defects, and behavioral abnormalities in tau ${ }_{45--230}$ transgenic mice. Neuroscience 275, 322-339. doi: 10.1016/j.neuroscience.2014.06.017

LeBlanc, A. C., Ramcharitar, J., Afonso, V., Hamel, E., Bennett, D. A., Pakavathkumar, P., et al. (2014). Caspase-6 activity in the CA1 region of the hippocampus induces age-dependent memory impairment. Cell. Death Differ. 21, 696-706. doi: 10.1038/cdd.2013.194

Li, J., Liu, D., Sun, L., Lu, Y., and Zhang, Z. (2012). Advanced glycation end products and neurodegenerative diseases: mechanisms and perspective. J. Neurol. Sci. 317, 1-5. doi: 10.1016/j.jns.2012.02.018

Li, K., Ito, H., Kominami, E., and Hirano, A. (1993). Abnormal distribution of cathepsin proteinases and endogenous inhibitors (cystatins) in the hippocampus of patients with Alzheimer's disease, parkinsonism-dementia complex on Guam, and senile dementia and in the aged. Virchows Arch. A Pathol. Anat. Histopathol. 423, 185-194. doi: 10.1007/BF01614769

Liu, F., and Gong, C. X. (2008). Tau exon 10 alternative splicing and tauopathies. Mol. Neurodegener. 3:8. doi: 10.1186/1750-1326-3-8

Liu, F., Grundke-Iqbal, I., Iqbal, K., and Gong, C. X. (2005). Contributions of protein phosphatases PP1, PP2A, PP2B and PP5 to the regulation of tau phosphorylation. Eur. J. Neurosci. 22, 1942-1950. doi: 10.1111/j.1460-9568. 2005.04391.x

Liu, F., Iqbal, K., Grundke-Iqbal, I., Hart, G. W., and Gong, C. X. O. (2004). GlcNAcylation regulates phosphorylation of tau: a mechanism involved in Alzheimer's disease. Proc. Natl. Acad. Sci. U. S. A. 101, 10804-10809. doi: 10.1073/pnas.0400348101

Liu, K., Liu, Y., Li, L., Qin, P., Iqbal, J., Deng, Y., et al. (2016). Glycation alter the process of Tau phosphorylation to change Tau isoforms aggregation property. Biochim. Biophys. Acta 1862, 192-201. doi: 10.1016/j.bbadis.2015.12.002

Lu, M., and Kosik, K. S. (2001). Competition for microtubule-binding with dual expression of tau missense and splice isoforms. Mol. Biol. Cell. 12, 171-184. doi: $10.1091 / \mathrm{mbc} \cdot 12.1 .171$

Luo, H. B., Xia, Y. Y., Shu, X. J., Liu, Z. C., Feng, Y., Liu, X. H., et al. (2014). SUMOylation at K340 inhibits tau degradation through deregulating its phosphorylation and ubiquitination. Proc. Natl. Acad. Sci. U. S. A. 111, 16586-16591. doi: 10.1073/pnas.1417548111

Maeda, S., Sahara, N., Saito, Y., Murayama, S., Ikai, A., and Takashima, A. (2006). Increased levels of granular tau oligomers: an early sign of brain aging and Alzheimer's disease. Neurosci. Res. 54, 197-201. doi: 10.1016/j.neures.2005.11. 009

Makrides, V., Massie, M. R., Feinstein, S. C., and Lew, J. (2004). Evidence for two distinct binding sites for tau on microtubules. Proc. Natl. Acad. Sci. U. S. A. 101, 6746-6751. doi: 10.1073/pnas.0400992101

Mandelkow, E. M., and Mandelkow, E. (2012). Biochemistry and cell biology of tau protein in neurofibrillary degeneration. Cold Spring Harb. Perspect. Med. 2:a006247. doi: 10.1101/cshperspect.a006247

Martin, L., Latypova, X., and Terro, F. (2011). Post-translational modifications of tau protein: implications for Alzheimer's disease. Neurochem. Int. 58, 458-471. doi: 10.1016/j.neuint.2010.12.023

Matsumoto, S. E., Motoi, Y., Ishiguro, K., Tabira, T., Kametani, F., Hasegawa, M., et al. (2015). The twenty-four KDa C-terminal tau fragment increases with aging in tauopathy mice: implications of prion-like properties. Hum. Mol. Genet. 24, 6403-6416. doi: 10.1093/hmg/ddv351

Matthews-Roberson, T. A., Quintanilla, R. A., Ding, H., and Johnson, G. V. (2008). Immortalized cortical neurons expressing caspase-cleaved tau are sensitized to endoplasmic reticulum stress induced cell death. Brain Res. 1234, 206-212. doi: 10.1016/j.brainres.2008.07.111

Merezhko, M., Uronen, R. L., and Huttunen, H. J. (2020). The Cell Biology of Tau Secretion. Front. Mol. Neurosci. 13:569818. doi: 10.3389/fnmol.2020.5 69818

Min, S. W., Chen, X., Tracy, T. E., Li, Y., Zhou, Y., Wang, C., et al. (2015). Critical role of acetylation in tau-mediated neurodegeneration and cognitive deficits. Nat. Med. 21, 1154-1162. doi: 10.3389/fnmol.2020.569818

Min, S. W., Cho, S. H., Zhou, Y., Schroeder, S., Haroutunian, V., Seeley, W. W., et al. (2010). Acetylation of tau inhibits its degradation and contributes to tauopathy. Neuron 67, 953-966. doi: 10.1016/j.neuron.2010.08.044

Miyamoto, K., Kowalska, A., Hasegawa, M., Tabira, T., Takahashi, K., Araki, W., et al. (2001). Familial frontotemporal dementia and parkinsonism with a novel 
mutation at an intron 10+11-splice site in the tau gene. Ann. Neurol. 50, 117-120. doi: 10.1002/ana.1083

Momeni, P., Pittman, A., Lashley, T., Vandrovcova, J., Malzer, E., Luk, C., et al. (2009). Clinical and pathological features of an Alzheimer's disease patient with the MAPT $\triangle$ K280 mutation. Neurobiol. Aging 30, 388-393. doi: 10.1016/j. neurobiolaging.2007.07.013

Moreno-Castillo, E., Álvarez-Ginarte, Y. M., Valdés-Tresanco, M. E., MonteroCabrera, L. A., Moreno, E., and Valiente, P. A. (2020). Understanding the disrupting mechanism of the Tau aggregation motif "306 VQIVYK311 " by phenylthiazolyl-hydrazides inhibitors. J. Mol. Recognit. 33:e2848. doi: 10.1002/ jmr.2848

Mukhopadhyay, D., and Riezman, H. (2007). Proteasome-independent functions of ubiquitin in endocytosis and signaling. Science 315, 201-205. doi: 10.1126/ science. 1127085

Munari, F., Barracchia, C. G., Parolini, F., Tira, R., Bubacco, L., Assfalg, M., et al. (2020). Semisynthetic Modification of Tau Protein with Di-Ubiquitin Chains for Aggregation Studies. Int. J. Mol. Sci. 21:4400. doi: 10.3390/ijms21124400

Nacharaju, P, Ko, L, and Yen, S. H. (1997). Characterization of in vitro glycation sites of tau. J. Neurochem. 69, 1709-19. doi: 10.1046/j.1471-4159.1997. 69041709.x

Nathan, J. A, Kim, H. T, Ting, L, Gygi, S. P, Goldberg, A. L. (2013). Why do cellular proteins linked to K63-polyubiquitin chains not associate with proteasomes? EMBO J. 32, 552-65. doi: 10.1038/emboj.2012.354

Ozcelik, S, Sprenger, F, Skachokova, Z, Fraser, G, Abramowski, D, Clavaguera, F, et al. (2016). Co-expression of truncated and full-length tau induces severe neurotoxicity. Mol. Psychiatry 21, 1790-1798. doi: 10.1038/mp.2015.228

Park, S, and Ferreira, A. (2005). The generation of a $17 \mathrm{kDa}$ neurotoxic fragment: an alternative mechanism by which tau mediates beta-amyloid-induced neurodegeneration. J. Neurosci. 25, 5365-5375. doi: 10.1523/JNEUROSCI. 1125-05.2005

Park, S, Lee, J. H, Jeon, J. H,and Lee, M. J. (2018). Degradation or aggregation: the ramifications of post-translational modifications on tau. BMB Rep. 51, 265-273. doi: 10.5483/BMBRep.2018.51.6.077

Pernègre, C, Duquette, A, and Leclerc, N. (2019). Tau Secretion: good and Bad for Neurons. Front Neurosci. 13:649. doi: 10.3389/fnins.2019.00649

Perry, G, Kawai, M, Tabaton, M, Onorato, M, Mulvihill, P, Richey, P, et al. (1991). Neuropil threads of Alzheimer's disease show a marked alteration of the normal cytoskeleton. J. Neurosci. 11, 1748-55. doi: 10.1523/JNEUROSCI.11-06-01748. 1991

Pevalova, M, Filipcik, P, Novak, M, Avila, J, and Iqbal, K. (2006). Post-translational modifications of tau protein. Bratisl. Lek. Listy 107, 346-53.

Poepsel, S, Sprengel, A, Sacca, B, Kaschani, F, Kaiser, M, Gatsogiannis, C, et al. (2015). Determinants of amyloid fibril degradation by the PDZ protease HTRA1. Nat. Chem. Biol. 11, 862-9. doi: 10.1038/nchembio.1931

Quinn, J. P, Corbett, N. J, Kellett, K. A. B, and Hooper, N. M. (2018). Tau Proteolysis in the Pathogenesis of Tauopathies: neurotoxic Fragments and Novel Biomarkers. J. Alzheimer. Dis. 63, 13-33. doi: 10.3233/JAD-170959

Quintanilla, R. A, Matthews-Roberson, T. A, Dolan, P. J, and Johnson, G. V (2009). Caspase-cleaved tau expression induces mitochondrial dysfunction in immortalized cortical neurons: implications for the pathogenesis of Alzheimer disease. J. Biol. Chem. 284, 18754-18766. doi: 10.1074/jbc.M80890 8200

Ramcharitar, J, Albrecht, S, Afonso, V. M, Kaushal, V, Bennett, D. A, and Leblanc, A. C. et al. (2013). Cerebrospinal fluid tau cleaved by caspase- 6 reflects brain levels and cognition in aging and Alzheimer disease. J. Neuropathol. Exp. Neurol. 72, 824-832. doi: 10.1097/NEN.0b013e3182a0a39f

Ramsden, M, Kotilinek, L, Forster, C, Paulson, J, McGowan, E, SantaCruz, K, et al. (2005). Age-dependent neurofibrillary tangle formation, neuron loss, and memory impairment in a mouse model of human tauopathy (P301L). J. Neurosci. 25, 10637-47. doi: 10.1523/JNEUROSCI.3279-05.2005

Rossi, G, and Tagliavini, F. (2015). Frontotemporal lobar degeneration: old knowledge and new insight into the pathogenetic mechanisms of tau mutations. Front. Aging Neurosci. 7:192. doi: 10.3389/fnagi.2015.00192

Rungratanawanich, W, Qu, Y, Wang, X, Essa, M. M, and Song, B. J. (2021). Advanced glycation end products (AGEs) and other adducts in aging-related diseases and alcohol-mediated tissue injury. Exp. Mol. Med. 53, 168-188. doi: 10.1038/s12276-021-00561-7
Santacruz, K, Lewis, J, Spires, T, Paulson, J, Kotilinek, L, Ingelsson, M, et al. (2005). Tau suppression in a neurodegenerative mouse model improves memory function. Science 309, 476-81. doi: 10.1126/science.1113694

Sasaki, N, Fukatsu, R, Tsuzuki, K, Hayashi, Y, Yoshida, T, Fujii, N, et al. (1998). Advanced glycation end products in Alzheimer's disease and other neurodegenerative diseases. Am. J. Pathol. 153, 1149-55. doi: 10.1016/S00029440(10)65659-3

Sayas, C. L, Medina, M, Cuadros, R, Ollá, I, García, E, Pérez, M, et al. (2019). Role of tau N-terminal motif in the secretion of human tau by End Binding proteins. PLoS One 14:e0210864. doi: 10.1371/journal.pone.0210864

Schneider, A, Biernat, J, von Bergen, M, Mandelkow, E, and Mandelkow, E. M., (1999). Phosphorylation that detaches tau protein from microtubules (Ser262, Ser214) also protects it against aggregation into Alzheimer paired helical filaments. Biochemistry 38, 3549-58. doi: 10.1021/bi981874p

Schueller, E, Paiva, I, Blanc, F, Wang, X. L, Cassel, J. C, Boutillier, A. L, et al. (2020). Dysregulation of histone acetylation pathways in hippocampus and frontal cortex of Alzheimer's disease patients. Eur. Neuropsychopharmacol. 33, 101-116. doi: 10.1016/j.euroneuro.2020.01.015

Shi, Y, Murzin, A. G, Falcon, B, Epstein, A, Machin, J, Tempest, P, et al. (2021). Cryo-EM structures of tau filaments from Alzheimer's disease with PET ligand APN-1607. Acta Neuropathol. 141, 697-708. doi: 10.1007/s00401-02102294-3

Shoji, M. (2019). Cerebrospinal Fluid and Plasma Tau as a Biomarker for Brain Tauopathy. Adv. Exp. Med. Biol. 1184, 393-405. doi: 10.1007/978-981-32-9358$8 \quad 29$

Siklos, M, BenAissa, M,and Thatcher, G. R (2015). Cysteine proteases as therapeutic targets: does selectivity matter? A systematic review of calpain and cathepsin inhibitors. Acta Pharm. Sin. B 5, 506-19. doi: 10.1016/j.apsb.2015.08.001

Šimić, G, Babić Leko, M, Wray, S, Harrington, C, Delalle, I, Jovanov-Milošević, $\mathrm{N}$, et al. (2016). Tau Protein Hyperphosphorylation and Aggregation in Alzheimer's Disease and Other Tauopathies, and Possible Neuroprotective Strategies. Biomolecules 6:6. doi: 10.3390/biom6010006

Simm, A, Müller, B, Nass, N, Hofmann, B, Bushnaq, H, Silber, R. E, et al. (2015). Protein glycation - Between tissue aging and protection. Exp. Gerontol. 68, 71-5. doi: 10.1016/j.exger.2014.12.013

Sokolow, S, Henkins, K. M, Bilousova, T, Gonzalez, B, Vinters, H. V, Miller, C. A, et al. (2015). "Pre-synaptic C-terminal truncated tau is released from cortical synapses in Alzheimer's disease." J. Neurochem. 133, 368-79. doi: 10.1111/jnc. 12991

Sontag, E, Nunbhakdi-Craig, V, Lee, G, Brandt, R, Kamibayashi, C, Kuret, J, et al. (1999). Molecular interactions among protein phosphatase 2A, tau, and microtubules. Implications for the regulation of tau phosphorylation and the development of tauopathies. J. Biol. Chem. 274, 25490-8. doi: 10.1074/jbc.274. 36.25490

Spillantini, M. G, and Goedert, M. (2000). Tau mutations in familial frontotemporal dementia. Brain 123, 857-9. doi: 10.1093/brain/123.5.857

Spillantini, M. G, Murrell, J. R, Goedert, M, Farlow, M. R, Klug, A, Ghetti, B. et al. (1998). Mutation in the tau gene in familial multiple system tauopathy with presenile dementia. Proc. Natl. Acad. Sci. U. S. A. 95, 7737-41. doi: 10.1073/ pnas.95.13.7737

Strang, K. H, Golde, T. E, and Giasson, B. I., (2019). MAPT mutations, tauopathy, and mechanisms of neurodegeneration. Lab. Invest. 99, 912-928. doi: 10.1038/ s41374-019-0197- $x$

Stringer, D. K, and Piper, R. C., (2011). Terminating protein ubiquitination: hasta la vista, ubiquitin. Cell Cycle. 10, 3067-71. doi: 10.4161/cc.10.18.17191

Suo, Z, Wu, M, Citron, B. A, Palazzo, R. E, and Festoff, B. W., (2003). Rapid tau aggregation and delayed hippocampal neuronal death induced by persistent thrombin signaling. J. Biol. Chem. 278, 37681-9. doi: 10.1074/jbc.M301406200

Suzuki K, Hata S, Kawabata Y, and Sorimachi H. (2004). Structure, activation, and biology of calpain. Diabetes 53, S12-8. doi: 10.2337/diabetes.53.2007.S12

Tennstaedt A, Pöpsel S, Truebestein L, Hauske P, Brockmann A, Schmidt N, et al. (2012). Human high temperature requirement serine protease Al (HTRA1) degrades tau protein aggregates. J. Biol. Chem. 287, 20931-41. doi: 10.1074/jbc. M111.316232

Tracy, T. E., and Gan, L. (2017). Acetylated tau in Alzheimer's disease: an instigator of synaptic dysfunction underlying memory loss: increased levels of acetylated tau blocks the postsynaptic signaling required for 
plasticity and promotes memory deficits associated with tauopathy. Bioessays 39:10.1002/bies.201600224. doi: 10.1002/bies.201600224

Tsuboi, Y., Wszolek, Z. K., Graff-Radford, N. R., Cookson, N., and Dickson, D. W. (2003). Tau pathology in the olfactory bulb correlates with Braak stage, Lewy body pathology and apolipoprotein epsilon4. Neuropathol. Appl. Neurobiol. 29, 503-510. doi: 10.1046/j.1365-2990.2003.00453.x

Tuerde, D., Kimura, T., Miyasaka, T., Furusawa, K., Shimozawa, A., Hasegawa, M., et al. (2018). Isoform-independent and -dependent phosphorylation of microtubule-associated protein tau in mouse brain during postnatal development. J. Biol. Chem. 293, 1781-1793. doi: 10.1074/jbc.M117.798918

Turgeon, V. L., Lloyd, E. D., Wang, S., Festoff, B. W., and Houenou, L. J. (1998). Thrombin perturbs neurite outgrowth and induces apoptotic cell death in enriched chick spinal motoneuron cultures through caspase activation. J. Neurosci. 18, 6882-6891. doi: 10.1523/JNEUROSCI.18-17-06882.1998

Uberti, D., Rizzini, C., Spano, P. F., and Memo, M. (1997). Characterization of tau proteins in human neuroblastoma SH-SY5Y cell line. Neurosci. Lett. 235, 149-153. doi: 10.1016/S0304-3940(97)00715-5

Van Opdenbosch, N., and Lamkanfi, M. (2019). Caspases in Cell Death, Inflammation, and Disease. Immunity 50, 1352-1364. doi: 10.1016/j.immuni. 2019.05.020

Van Swieten, J. C., Bronner, I. F., Azmani, A., Severijnen, L. A., Kamphorst, W., Ravid, R., et al. (2007). The $\triangle \mathrm{K} 280$ mutation in MAP tau favors exon 10 skipping in vivo. J. Neuropathol. Exp. Neurol. 66, 17-25. doi: 10.1097/nen. 0b013e31802c39a4

Wagner, U., Utton, M., Gallo, J. M., and Miller, C. C. (1996). Cellular phosphorylation of tau by GSK-3 beta influences tau binding to microtubules and microtubule organization. J. Cell. Sci. 109, 1537-1543. doi: 10.1242/jcs.109. 6.1537

Wang, J. Z., Gong, C. X., Zaidi, T., Grundke-Iqbal, I., and Iqbal, K. (1995). Dephosphorylation of Alzheimer paired helical filaments by protein phosphatase-2A and -2B. J. Biol. Chem. 270, 4854-4860. doi: 10.1074/jbc.270. 9.4854

Wang, J. Z., Xia, Y. Y., Grundke-Iqbal, I., and Iqbal, K. (2013). Abnormal hyperphosphorylation of tau: sites, regulation, and molecular mechanism of neurofibrillary degeneration. J. Alzheimer. Dis. 33, S123-S139. doi: 0.3233/ JAD-2012-129031

Wang, Y. P., Biernat, J., Pickhardt, M., Mandelkow, E., and Mandelkow, E. M. (2007). Stepwise proteolysis liberates tau fragments that nucleate the Alzheimer-like aggregation of full-length tau in a neuronal cell model. Proc. Natl. Acad. Sci. U. S. A. 104, 10252-10257. doi: 10.1073/pnas.0703676104

Wang, Y., Martinez-Vicente, M., Krüger, U., Kaushik, S., Wong, E., Mandelkow, E. M., et al. (2009). Tau fragmentation, aggregation and clearance: the dual role of lysosomal processing. Hum. Mol. Genet. 18, 4153-4170. doi: 10.1093/hmg/ ddp367

Wang, Z. H., Liu, P., Liu, X., Manfredsson, F. P., Sandoval, I. M., Yu, S. P., et al. (2017). Delta-Secretase Phosphorylation by SRPK2 Enhances Its Enzymatic Activity, Provoking Pathogenesis in Alzheimer's Disease. Mol Cell. 67, e812825. doi: 10.1016/j.molcel.2017.07.018

Weingarten, M. D., Lockwood, A. H., Hwo, S. Y., and Kirschner, M. W. (1975). A protein factor essential for microtubule assembly. Proc. Natl. Acad. Sci. U. S. A. 72, 1858-1862. doi: 10.1073/pnas.72.5.1858

Wille, H., Drewes, G., Biernat, J., and Mandelkow, E. M. (1992). E. Alzheimer-like paired helical filaments and antiparallel dimers formed from microtubuleassociated protein tau in vitro. J. Cell. Biol. 118, 573-584. doi: 10.1083/jcb.118. 3.573

Wischik, C. M., Novak, M., Edwards, P. C., Klug, A., Tichelaar, W., and Crowther, R. A. (1988). Structural characterization of the core of the paired helical filament of Alzheimer disease. Proc. Natl. Acad. Sci. U. S. A. 85, 4884-4888. doi: 10.1073/ pnas.85.13.4884

Wolfe, M. S. (2009). Tau mutations in neurodegenerative diseases. J. Biol. Chem. 284, 6021-6025. doi: 10.1074/jbc.R800013200

Wolfe, M. S. (2012). The role of tau in neurodegenerative diseases and its potential as a therapeutic target. Scientifica 2012:796024. doi: 10.6064/2012/796024

Wong, M. B., Goodwin, J., Norazit, A., Meedeniya, A. C., Richter-Landsberg, C., Gai, W. P., et al. (2013). SUMO-1 is associated with a subset of lysosomes in glial protein aggregate diseases. Neurotox. Res. 23, 1-21. doi: 10.1007/s12640012-9358-z

Yamada, K., Cirrito, J. R., Stewart, F. R., Jiang, H., Finn, M. B., Holmes, B. B., et al. (2011). In vivo microdialysis reveals age-dependent decrease of brain interstitial fluid tau levels in P301S human tau transgenic mice. J. Neurosci. 31, 13110-13117. doi: 10.1523/JNEUROSCI.2569-11 .2011

Yamashima, T. (2013). Reconsider Alzheimer's disease by the 'calpain-cathepsin hypothesis'-a perspective review. Prog. Neurobiol. 105, 1-23. doi: 10.1016/j. pneurobio.2013.02.004

Yang, L. S., and Ksiezak-Reding, H. (1995). Calpain-induced proteolysis of normal human tau and tau associated with paired helical filaments. Eur. J. Biochem. 233, 9-17. doi: 10.1111/j.1432-1033.1995.009_1.x

Yin, X., Wright, J., Wall, T., and Grammas, P. (2010). Brain endothelial cells synthesize neurotoxic thrombin in Alzheimer's disease. Am. J. Pathol. 176, 1600-1606. doi: 10.2353/ajpath.2010.090406

Yin, Y., Wang, Y., Gao, D., Ye, J., Wang, X., Fang, L., et al. (2016). Accumulation of human full-length tau induces degradation of nicotinic acetylcholine receptor $\alpha 4$ via activating calpain-2. Sci. Rep. 6:27283. doi: 10.1038/srep 27283

Yoshida, M. (2006). Cellular tau pathology and immunohistochemical study of tau isoforms in sporadic tauopathies. Neuropathology 26, 457-470. doi: 10.1111/j. 1440-1789.2006.00743.x

Yu, Y., Run, X., Liang, Z., Li, Y., Liu, F., Liu, Y., et al. (2009). Developmental regulation of tau phosphorylation, tau kinases, and tau phosphatases. J. Neurochem. 108, 1480-1494. doi:10.1111/j.1471-4159.2009.0 5882.x

Yuzwa, S. A., Cheung, A. H., Okon, M., McIntosh, L. P., and Vocadlo, D. J. O. - (2014). GlcNAc modification of tau directly inhibits its aggregation without perturbing the conformational properties of tau monomers. J. Mol. Biol. 426, 1736-1752. doi: 10.1016/j.jmb.2014.01.004

Yuzwa, S. A., Shan, X., Macauley, M. S., Clark, T., Skorobogatko, Y., Vosseller, K., et al. (2012). Increasing O-GlcNAc slows neurodegeneration and stabilizes tau against aggregation. Nat. Chem. Biol. 8, 393-399. doi: 10.1038/nchembio.797

Zhang, H., Zhang, Y. W., Chen, Y., Huang, X., Zhou, F., Wang, W., et al. (2012). Appoptosin is a novel pro-apoptotic protein and mediates cell death in neurodegeneration. J. Neurosci. 32, 15565-15576. doi: 10.1523/JNEUROSCI. 3668-12.2012

Zhang, Z., Kang, S. S., Liu, X., Ahn, E. H., Zhang, Z., He, L., et al. (2017). Asparagine endopeptidase cleaves $\alpha$-synuclein and mediates pathologic activities in Parkinson's disease. Nat. Struct. Mol. Biol. 24, 632-642. doi: 10.1038/nsmb.3433

Zhang, Z., Song, M., Liu, X., Kang, S. S., Kwon, I. S., Duong, D. M., et al. (2014). Cleavage of tau by asparagine endopeptidase mediates the neurofibrillary pathology in Alzheimer's disease. Nat. Med. 20, 1254-1262. doi: 10.1038/nm. 3700

Zhang, Z., Song, M., Liu, X., Su Kang, S., Duong, D. M., Seyfried, N. T., et al. (2015). Delta-secretase cleaves amyloid precursor protein and regulates the pathogenesis in Alzheimer's disease. Nat. Commun. 6:8762. doi: 10.1038/ ncomms 9762

Zhao, X., Kotilinek, L. A., Smith, B., Hlynialuk, C., Zahs, K., Ramsden, M., et al. (2016). Caspase-2 cleavage of tau reversibly impairs memory. Nat. Med. 22, 1268-1276. doi: 10.1038/nm.4199

Zhao, Y., Tseng, I. C., Heyser, C. J., Rockenstein, E., Mante, M., Adame, A., et al. (2015). Appoptosin-Mediated Caspase Cleavage of Tau Contributes to Progressive Supranuclear Palsy Pathogenesis. Neuron 87, 963-975. doi: 10. 1016/j.neuron.2015.08.020

Conflict of Interest: The authors declare that the research was conducted in the absence of any commercial or financial relationships that could be construed as a potential conflict of interest.

Publisher's Note: All claims expressed in this article are solely those of the authors and do not necessarily represent those of their affiliated organizations, or those of the publisher, the editors and the reviewers. Any product that may be evaluated in this article, or claim that may be made by its manufacturer, is not guaranteed or endorsed by the publisher.

Copyright (C) 2021 Boyarko and Hook. This is an open-access article distributed under the terms of the Creative Commons Attribution License (CC BY). The use, distribution or reproduction in other forums is permitted, provided the original author(s) and the copyright owner(s) are credited and that the original publication in this journal is cited, in accordance with accepted academic practice. No use, distribution or reproduction is permitted which does not comply with these terms. 